\title{
Long range and local air pollution: what can we learn from chemical speciation of particulate matter at paired sites?
}

Marco Pandolfi ${ }^{a, k}$, Dennis Mooibroek ${ }^{b}$, Philip Hopke ${ }^{c}$, Dominik van Pinxteren ${ }^{d}$, Xavier Querol

${ }^{a}$, Herrmann Hartmut ${ }^{d}$, Andrés Alastuey ${ }^{a}$, Olivier Favez ${ }^{e}$, Christoph Hüglin ${ }^{\dagger}$, Esperanza Perdrix ${ }^{g}$, Véronique Riffault ${ }^{g}$, Stéphane Sauvage ${ }^{g}$, Eric van der Swaluw ${ }^{b}$, Oksana Tarasova ${ }^{\mathrm{h}}$, and Augustin Colette ${ }^{\mathrm{e}}$

${ }^{a}$ Institute of Environmental Analysis and Water Research (IDAEA-CSIC), c/ Jordi-Girona 18-26, Barcelona, Spain

${ }^{b}$ Centre for Environmental Monitoring, National Institute of Public Health and the Environment (RIVM), A. van Leeuwenhoeklaan 9, P.O. Box 1, 3720 BA, Bilthoven, The Netherlands

${ }^{c}$ Center for Air Resources Engineering and Science, Clarkson University, Potsdam, NY, USA

${ }^{d}$ Leibniz Institute for Tropospheric Research (TROPOS), Atmospheric Chemistry Department (ACD), Permoserstr. 15, 04318 Leipzig, Germany

e National Institute for Industrial Environment and Risks (INERIS), Verneuil-en-Halatte, 60550, France

f Empa, Swiss Federal Laboratories for Materials Science and Technology, 8600 Dübendorf, Switzerland

${ }^{9}$ IMT Lille Douai, Univ. Lille, SAGE - Département Sciences de I'Atmosphère et Génie de I'Environnement, 59000 Lille, France

${ }^{\mathrm{h}}$ World Meteorological Organization, Research Department, Geneva, Switzerland

*Corresponding author: Marco Pandolfi (marco.pandolfi@idaea.csic.es) 
Table S1: Chemical $\mathrm{PM}_{10}$ data sampled at Barcelona (BCN; UB), Montseny (MSY; RB) and Montsec (MSA; CB) (Spain) and used in the PMF model (2010 - 2014). Specie concentrations are reported in $\mu \mathrm{g} / \mathrm{m}^{3}$.

\begin{tabular}{|c|c|c|c|c|c|c|c|c|c|c|}
\hline Chemical specie & \multicolumn{3}{|c|}{$\mathrm{BCN}$} & \multicolumn{3}{|c|}{ MSY } & \multicolumn{3}{|c|}{ MSA } & \\
\hline$\left[\mu \mathrm{g} / \mathrm{m}^{3}\right]$ & mean & SD & median & mean & SD & median & mean & SD & median & \\
\hline $\mathrm{PM}_{10}$ & 24.5719 & 10.1681 & 23.0796 & 16.3678 & 9.2712 & 15.0258 & 9.3843 & 7.8660 & 7.4151 & \\
\hline Al & 0.2611 & 0.2306 & 0.1880 & 0.2773 & 0.5118 & 0.1441 & 0.2493 & 0.5356 & 0.1176 & \\
\hline $\mathrm{Ca}$ & 0.6699 & 0.4680 & 0.5431 & 0.2887 & 0.3444 & 0.1937 & 0.3517 & 0.4216 & 0.2049 & \\
\hline K & 0.2129 & 0.1471 & 0.1915 & 0.1420 & 0.1432 & 0.1060 & 0.1105 & 0.1508 & 0.0863 & \\
\hline $\mathrm{Na}$ & 0.7847 & 0.6495 & 0.6153 & 0.3048 & 0.2704 & 0.2162 & 0.1711 & 0.1729 & 0.1143 & \\
\hline $\mathrm{Mg}$ & 0.1661 & 0.0999 & 0.1389 & 0.1013 & 0.1163 & 0.0724 & 0.0760 & 0.1149 & 0.0476 & \\
\hline $\mathrm{Fe}$ & 0.5097 & 0.2823 & 0.4394 & 0.2052 & 0.3401 & 0.1022 & 0.1368 & 0.2874 & 0.0671 & \\
\hline$M n$ & 0.0101 & 0.0061 & 0.0088 & 0.0040 & 0.0044 & 0.0029 & 0.0041 & 0.0058 & 0.0026 & \\
\hline $\mathrm{Ti}$ & 0.0183 & 0.0135 & 0.0148 & 0.0149 & 0.0267 & 0.0076 & 0.0148 & 0.0342 & 0.0066 & \\
\hline V & 0.0056 & 0.0043 & 0.0046 & 0.0020 & 0.0018 & 0.0015 & 0.0012 & 0.0015 & 0.0007 & \\
\hline $\mathrm{Ni}$ & 0.0027 & 0.0023 & 0.0021 & 0.0012 & 0.0015 & 0.0009 & 0.0006 & 0.0011 & 0.0004 & \\
\hline $\mathrm{Cu}$ & 0.0189 & 0.0119 & 0.0163 & 0.0029 & 0.0018 & 0.0026 & 0.0011 & 0.0010 & 0.0009 & \\
\hline As & 0.0004 & 0.0002 & 0.0004 & 0.0002 & 0.0001 & 0.0002 & 0.0001 & 0.0001 & 0.0001 & \\
\hline $\mathbf{R b}$ & 0.0005 & 0.0003 & 0.0004 & 0.0004 & 0.0005 & 0.0002 & 0.0003 & 0.0006 & 0.0002 & \\
\hline Sr & 0.0024 & 0.0020 & 0.0020 & 0.0013 & 0.0021 & 0.0008 & 0.0016 & 0.0025 & 0.0009 & \\
\hline $\mathrm{Sb}$ & 0.0024 & 0.0016 & 0.0020 & 0.0003 & 0.0002 & 0.0003 & 0.0001 & 0.0002 & 0.0000 & \\
\hline $\mathbf{P b}$ & 0.0083 & 0.0114 & 0.0061 & 0.0023 & 0.0019 & 0.0020 & 0.0012 & 0.0009 & 0.0009 & \\
\hline $\mathrm{SO}_{4}{ }^{2-}$ & 2.6322 & 1.6846 & 2.2756 & 1.8946 & 1.4842 & 1.6141 & 1.3343 & 1.1454 & 1.0266 & \\
\hline $\mathrm{NO}_{3}{ }^{-}$ & 2.4192 & 2.1829 & 1.7304 & 1.0455 & 1.0382 & 0.7587 & 0.7540 & 0.8747 & 0.4910 & \\
\hline $\mathrm{Cl}^{-}$ & 0.6844 & 0.7647 & 0.4260 & 0.2488 & 0.2979 & 0.1662 & 0.1382 & 0.1762 & 0.1022 & \\
\hline $\mathrm{NH}_{4}{ }^{+}$ & 0.7736 & 0.8742 & 0.4737 & 0.4976 & 0.4713 & 0.3674 & 0.4482 & 0.4605 & 0.2983 & \\
\hline EC & 1.1545 & 0.6589 & 1.0129 & 0.2318 & 0.1291 & 0.2089 & 0.1071 & 0.0811 & 0.0930 & \\
\hline OC & 2.9088 & 1.4308 & 2.6011 & 1.8983 & 0.8589 & 1.7894 & 1.5188 & 0.8708 & 1.4112 & \\
\hline
\end{tabular}


Table S2: Chemical $\mathrm{PM}_{10}$ data sampled at Zurich (ZUE; UB) and Payerne (PAY; RB) (Switzerland) and used in the PMF model (2008-2009). Specie concentrations are reported in $\mu \mathrm{g} / \mathrm{m}^{3}$.

\begin{tabular}{|c|c|c|c|c|c|c|c|}
\hline Chemical specie & \multicolumn{3}{|c|}{ ZUE } & \multicolumn{3}{|c|}{ PAY } & \\
\hline$\left[\mu \mathrm{g} / \mathrm{m}^{3}\right]$ & mean & SD & median & mean & SD & median & \\
\hline $\mathrm{PM}_{10}$ & 20.41303 & 11.52026 & 15.77000 & 18.45809 & 11.84675 & 14.90000 & \\
\hline$O C$ & 3.70135 & 2.14696 & 3.09000 & 3.51303 & 2.23133 & 3.10000 & \\
\hline EC & 1.26427 & 0.74653 & 1.06000 & 0.66292 & 0.46896 & 0.55000 & \\
\hline $\mathrm{NO}_{3}^{-}$ & 3.78748 & 4.30968 & 1.98800 & 3.80962 & 4.64906 & 1.77700 & \\
\hline $\mathrm{SO}_{4}{ }^{2-}$ & 2.36776 & 1.53784 & 1.99100 & 1.91618 & 1.27660 & 1.68200 & \\
\hline $\mathrm{Na}^{+}$ & 0.15094 & 0.16235 & 0.10100 & 0.12454 & 0.12452 & 0.09200 & \\
\hline $\mathrm{NH}_{4}{ }^{+}$ & 1.61817 & 1.54775 & 1.06000 & 1.57806 & 1.58461 & 0.92600 & \\
\hline $\mathrm{K}^{+}$ & 0.20227 & 0.19533 & 0.12400 & 0.17213 & 0.16706 & 0.11100 & \\
\hline $\mathbf{M g}_{2}{ }^{+}$ & 0.02904 & 0.02118 & 0.02400 & 0.02048 & 0.01686 & 0.01800 & \\
\hline $\mathrm{Ca}_{2}{ }^{+}$ & 0.31661 & 0.28609 & 0.19600 & 0.17528 & 0.13329 & 0.13800 & \\
\hline Al & 0.07053 & 0.06501 & 0.04381 & 0.06521 & 0.06854 & 0.04636 & \\
\hline Ti & 0.00195 & 0.00133 & 0.00158 & 0.00171 & 0.00155 & 0.00124 & \\
\hline $\mathbf{V}$ & 0.00067 & 0.00046 & 0.00058 & 0.00056 & 0.00052 & 0.00039 & \\
\hline $\mathrm{Cr}$ & 0.00198 & 0.00161 & 0.00154 & 0.00066 & 0.00071 & 0.00048 & \\
\hline$M n$ & 0.00537 & 0.00318 & 0.00445 & 0.00264 & 0.00165 & 0.00229 & \\
\hline $\mathrm{Fe}$ & 0.46942 & 0.27748 & 0.41346 & 0.11914 & 0.07740 & 0.09687 & \\
\hline $\mathrm{Cu}$ & 0.02069 & 0.01165 & 0.01809 & 0.00415 & 0.00272 & 0.00338 & \\
\hline $\mathrm{Zn}$ & 0.02849 & 0.02162 & 0.02359 & 0.01958 & 0.01611 & 0.01735 & \\
\hline Ga & 0.00016 & 0.00011 & 0.00012 & 0.00008 & 0.00007 & 0.00006 & \\
\hline As & 0.00052 & 0.00106 & 0.00032 & 0.00050 & 0.00058 & 0.00037 & \\
\hline $\mathbf{R b}$ & 0.00049 & 0.00042 & 0.00035 & 0.00056 & 0.00040 & 0.00051 & \\
\hline $\mathrm{Sr}$ & 0.00076 & 0.00062 & 0.00054 & 0.00051 & 0.00048 & 0.00037 & \\
\hline$Y$ & 0.00004 & 0.00003 & 0.00003 & 0.00003 & 0.00003 & 0.00002 & \\
\hline Mo & 0.00116 & 0.00070 & 0.00099 & 0.00024 & 0.00017 & 0.00022 & \\
\hline Cd & 0.00012 & 0.00008 & 0.00010 & 0.00009 & 0.00007 & 0.00007 & \\
\hline Sb & 0.00225 & 0.00129 & 0.00192 & 0.00059 & 0.00040 & 0.00053 & \\
\hline $\mathrm{Ba}$ & 0.00370 & 0.00228 & 0.00310 & 0.00162 & 0.00188 & 0.00116 & \\
\hline La & 0.00008 & 0.00005 & 0.00007 & 0.00005 & 0.00005 & 0.00004 & \\
\hline $\mathrm{Ce}$ & 0.00014 & 0.00008 & 0.00013 & 0.00008 & 0.00007 & 0.00006 & \\
\hline Nd & 0.00004 & 0.00003 & 0.00003 & 0.00003 & 0.00003 & 0.00002 & \\
\hline $\mathrm{Pb}$ & 0.00533 & 0.00387 & 0.00396 & 0.00383 & 0.00311 & 0.00298 & \\
\hline
\end{tabular}


Table S3: Chemical $\mathrm{PM}_{2.5}$ data sampled at Schiedam (SCH; UB) and Hellendoorn (HEL; RB) (The Netherlands) and used in the PMF model (2007-2008). The concentration of major elements is reported in $\mathrm{mg} / \mathrm{m}^{3}$ and the concentration of trace elements in $\mathrm{ng} / \mathrm{m}^{3}$.

\begin{tabular}{|c|c|c|c|c|}
\hline \multirow{2}{*}{$\begin{array}{c}\text { Chemical specie } \\
{\left[\mu \mathrm{g} / \mathrm{m}^{3}\right]}\end{array}$} & \multicolumn{2}{|c|}{$\mathrm{SCH}$} & \multicolumn{2}{|c|}{ HEL } \\
\hline & mean & SD & mean & SD \\
\hline $\mathbf{P M}_{2.5}$ & 17.2 & 11.6 & 14.0 & 6.9 \\
\hline $\mathrm{OC}$ & 2.1 & 1.1 & 2.0 & 0.8 \\
\hline EC & 2.2 & 1.6 & 1.7 & 1.1 \\
\hline $\mathrm{NH}_{4}{ }^{+}$ & 1.2 & 1.5 & 1.6 & 1.4 \\
\hline $\mathrm{NO}_{3}^{-}$ & 2.8 & 3.4 & 3.7 & 3.1 \\
\hline $\mathrm{SO}_{4}{ }^{2-}$ & 2.6 & 1.4 & 2.6 & 1.8 \\
\hline $\mathrm{Cl}$ & 0.3 & 0.3 & 0.3 & 0.3 \\
\hline$\left[\mathrm{ng} / \mathrm{m}^{3}\right]$ & & & & \\
\hline Al & 61.9 & 131.7 & 35.6 & 74.3 \\
\hline As & 0.7 & 0.6 & 0.4 & 0.4 \\
\hline $\mathrm{Ba}$ & 11.0 & 56.3 & 5.5 & 16.5 \\
\hline $\mathrm{Ca}$ & 87.5 & 76.8 & 61.7 & 55.8 \\
\hline $\mathrm{Cd}$ & 0.3 & 0.3 & 0.3 & 0.2 \\
\hline Co & 0.3 & 0.2 & 0.2 & 0.1 \\
\hline $\mathrm{Cr}$ & 2.9 & 1.3 & 2.7 & 1.2 \\
\hline $\mathrm{Cu}$ & 5.5 & 9.9 & 2.5 & 3.3 \\
\hline $\mathrm{Fe}$ & 115.9 & 117.5 & 71.5 & 76.5 \\
\hline K & 134.2 & 529.5 & 84.0 & 124.7 \\
\hline Mg & 64.9 & 86.9 & 44.6 & 33.0 \\
\hline$M n$ & 4.0 & 3.4 & 2.6 & 2.3 \\
\hline Mo & 0.6 & 0.4 & 0.5 & 0.4 \\
\hline $\mathrm{Na}$ & 339.9 & 311.4 & 173.8 & 201.0 \\
\hline $\mathrm{Ni}$ & 5.4 & 4.0 & 1.9 & 1.2 \\
\hline $\mathbf{P}$ & 90.0 & 37.7 & 80.1 & 36.3 \\
\hline $\mathbf{P b}$ & 9.1 & 11.9 & 8.3 & 12.5 \\
\hline $\mathbf{S b}$ & 1.0 & 1.0 & 0.6 & 0.5 \\
\hline Se & 2.7 & 5.8 & 0.8 & 0.6 \\
\hline $\mathbf{S i}$ & 93.2 & 171.3 & 84.5 & 12.5 \\
\hline Sn & 4.2 & 11.2 & 0.9 & 0.8 \\
\hline $\mathrm{Sr}$ & 2.2 & 12.4 & 0.9 & 2.2 \\
\hline $\mathrm{Ti}$ & 2.5 & 2.9 & 1.5 & 2.0 \\
\hline $\mathbf{V}$ & 9.0 & 7.7 & 2.0 & 1.9 \\
\hline$Z n$ & 95.3 & 36.7 & 90.5 & 31.7 \\
\hline
\end{tabular}


Table S4: Chemical $\mathrm{PM}_{10}$ data sampled at Lens (LENS; UB) and Revin (REV; UB) (France) and used in the PMF model (2013-2014). Specie concentrations are reported in $\mu \mathrm{g} / \mathrm{m}^{3}$.

\begin{tabular}{|c|c|c|c|c|c|c|}
\hline Chemical specie & \multicolumn{3}{|c|}{ LENS } & \multicolumn{3}{|c|}{ REV } \\
\hline$\left[\mu \mathrm{g} / \mathrm{m}^{3}\right]$ & mean & SD & median & mean & SD & median \\
\hline $\mathbf{P M}_{10}$ & 20.5193 & 12.7126 & 16.0000 & 16.3038 & 9.6521 & 15.0000 \\
\hline EC & 0.6029 & 0.5851 & 0.4462 & 0.1742 & 0.0993 & 0.1537 \\
\hline$O C$ & 3.2052 & 2.6364 & 2.5157 & 2.1603 & 1.2652 & 1.8912 \\
\hline $\mathrm{Cl}$ - & 0.7378 & 0.9006 & 0.3797 & 0.3037 & 0.5599 & 0.0777 \\
\hline $\mathrm{NO}_{3}{ }^{-}$ & 5.0508 & 5.5206 & 2.5376 & 3.2520 & 4.0694 & 1.8593 \\
\hline $\mathrm{SO}_{4}{ }^{2-}$ & 2.3309 & 2.2417 & 1.6347 & 2.0347 & 1.7630 & 1.5002 \\
\hline $\mathrm{Na}^{+}$ & 0.6213 & 0.5690 & 0.4616 & 0.3787 & 0.4537 & 0.2097 \\
\hline $\mathrm{NH}_{4}{ }^{+}$ & 1.8741 & 2.1916 & 0.9945 & 1.2723 & 1.4603 & 0.7491 \\
\hline $\mathbf{K}^{+}$ & 0.1294 & 0.0982 & 0.1064 & 0.0503 & 0.0413 & 0.0399 \\
\hline $\mathrm{Mg}^{2+}$ & 0.0796 & 0.0640 & 0.0611 & 0.0408 & 0.0398 & 0.0278 \\
\hline MSA & 0.0728 & 0.0805 & 0.0503 & 0.0395 & 0.0508 & 0.0210 \\
\hline Levoglucosan & 0.1906 & 0.2729 & 0.0940 & 0.1003 & 0.0994 & 0.0719 \\
\hline Polisac & 0.0304 & 0.0684 & 0.0125 & 0.0117 & 0.0143 & 0.0061 \\
\hline Alcohols & 0.0239 & 0.0347 & 0.0116 & 0.0242 & 0.0305 & 0.0126 \\
\hline Al & 0.1566 & 0.2059 & 0.0993 & 0.1192 & 0.2519 & 0.0539 \\
\hline $\mathrm{Fe}$ & 0.2713 & 0.2757 & 0.1696 & 0.1675 & 0.1891 & 0.1108 \\
\hline $\mathrm{Ca}$ & 0.3682 & 0.4660 & 0.2266 & 0.2543 & 0.3906 & 0.1705 \\
\hline As & 0.0007 & 0.0008 & 0.0004 & 0.0007 & 0.0007 & 0.0004 \\
\hline Cd & 0.0002 & 0.0002 & 0.0002 & 0.0002 & 0.0003 & 0.0002 \\
\hline Co & 0.0002 & 0.0002 & 0.0001 & 0.0001 & 0.0001 & 0.0001 \\
\hline $\mathrm{Cu}$ & 0.0121 & 0.0167 & 0.0072 & 0.0045 & 0.0042 & 0.0032 \\
\hline La & 0.0002 & 0.0002 & 0.0001 & 0.0001 & 0.0001 & 0.0001 \\
\hline Mn & 0.0066 & 0.0060 & 0.0045 & 0.0061 & 0.0055 & 0.0042 \\
\hline $\mathrm{Pb}$ & 0.0087 & 0.0092 & 0.0056 & 0.0082 & 0.0061 & 0.0065 \\
\hline $\mathbf{R b}$ & 0.0005 & 0.0005 & 0.0003 & 0.0005 & 0.0004 & 0.0003 \\
\hline Sb & 0.0012 & 0.0011 & 0.0009 & 0.0006 & 0.0004 & 0.0005 \\
\hline Se & 0.0011 & 0.0009 & 0.0007 & 0.0012 & 0.0010 & 0.0010 \\
\hline $\mathbf{S r}$ & 0.0019 & 0.0019 & 0.0015 & 0.0014 & 0.0017 & 0.0011 \\
\hline $\mathrm{Ti}$ & 0.0102 & 0.0107 & 0.0069 & 0.0098 & 0.0121 & 0.0066 \\
\hline $\mathbf{Z n}$ & 0.0302 & 0.0374 & 0.0183 & 0.0363 & 0.0405 & 0.0246 \\
\hline
\end{tabular}




\section{- Source profiles from multi-site PMF for Spain.}

Figure $\mathrm{S} 1$ shows the chemical profiles of the sources detected at BCN, MSY and MSA from the multi-site PMF. A total of 7 common sources were identified at the three sites, namely:

- Heavy-Oil combustion (V-Ni), traced mainly by $\mathrm{V}, \mathrm{Ni}$ and $\mathrm{SO}_{4}{ }^{2-}$ and representing the direct emissions from heavy oil combustion sources, mostly shipping in the area under study during the period considered, but also long range transport. The sulfate associated with this source also includes primary sulfate from shipping (Ref.)

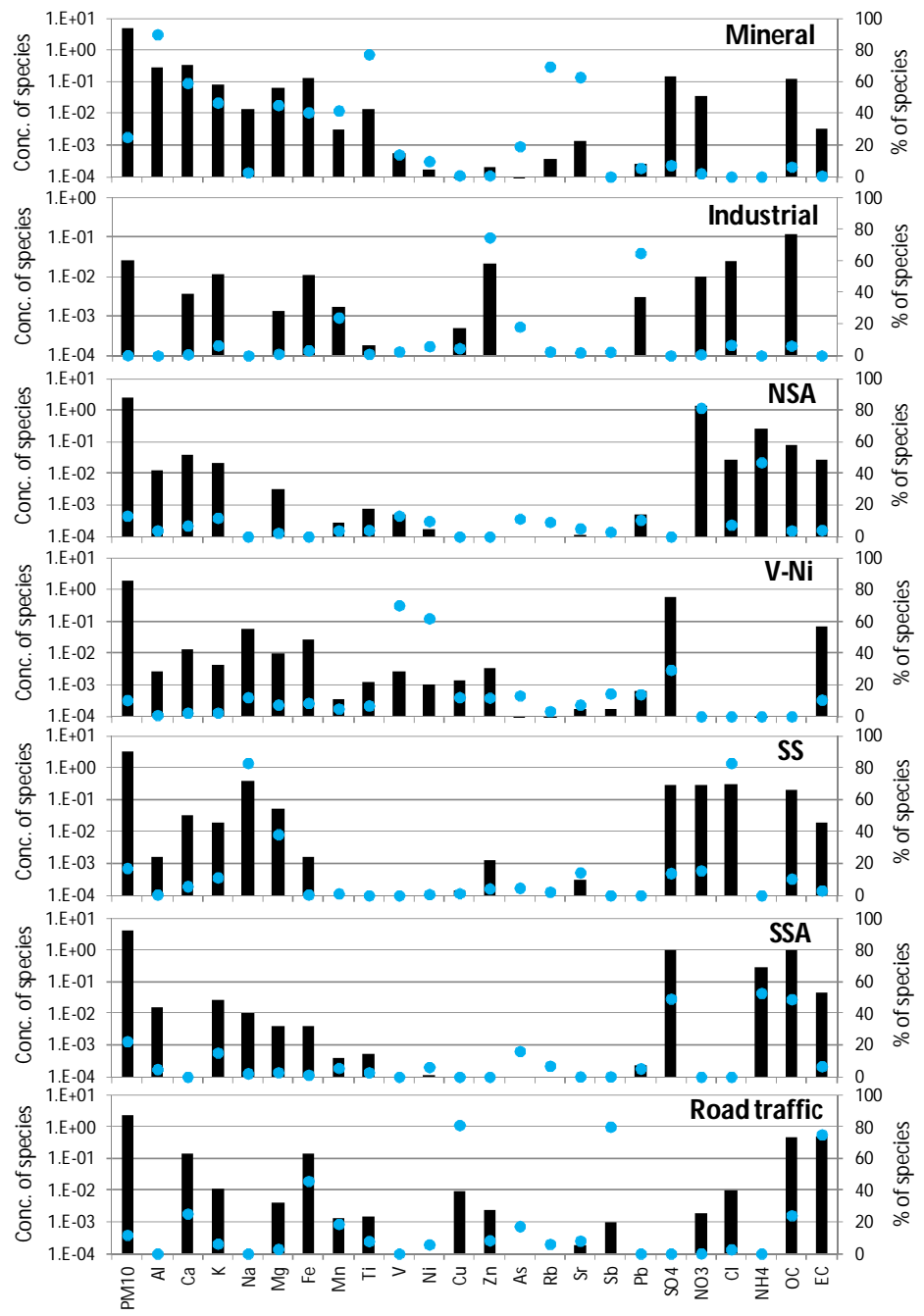

Figure S1: Chemical profiles of the sources detected at Barcelona (BCN; UB), Montseny (MSY; RB) and Montsec (MSA; CB) (Spain).

- Mineral (MM), traced by typical crustal elements such as $\mathrm{Al}, \mathrm{Ca}, \mathrm{Ti}, \mathrm{Rb}$, and $\mathrm{Sr}$;

- Sea salt (SS), traced by $\mathrm{Na}$ and $\mathrm{Cl}$ mainly with contributions from $\mathrm{SO}_{4}{ }^{2-}$ and $\mathrm{NO}_{3}{ }^{-}$ suggesting some aging of marine aerosols;

- Secondary sulfate (SSA), secondary inorganic source traced by $\mathrm{SO}_{4}{ }^{2-}$ and $\mathrm{NH}_{4}{ }^{+}$ with relatively high contents of $\mathrm{OC}$ which have been attributed to the condensation 
of semi-volatile compounds on the high specific surface area of ammonium sulfate particles (Amato et al., 2009);

- Primary Industrial (IND), traced by $\mathrm{Pb}$ and As representing mostly emissions from metallurgy;

- Primary Road Traffic (RT), traced mainly by EC, OC, Cu, Sb and Fe;

- Secondary nitrate (NSA), secondary inorganic source traced by $\mathrm{NO}_{3}{ }^{-}$and $\mathrm{NH}_{4}{ }^{+}$.

\section{- Source profiles from multi-site PMF for Switzerland.}

Figure S2 shows the chemical profiles of the sources from multi-site PMF for Switzerland. A total of 6 sources were identified at the two sites. A description of the sources is given below. The number and type of sources is the same as in Gianini et al. (2012):

- Primary Road Traffic (RT), explaining large fractions of EC, OC and of the road traffic related elements $(\mathrm{Mn}, \mathrm{Cr}, \mathrm{Fe}, \mathrm{Cu}, \mathrm{Mo}, \mathrm{Sb})$;

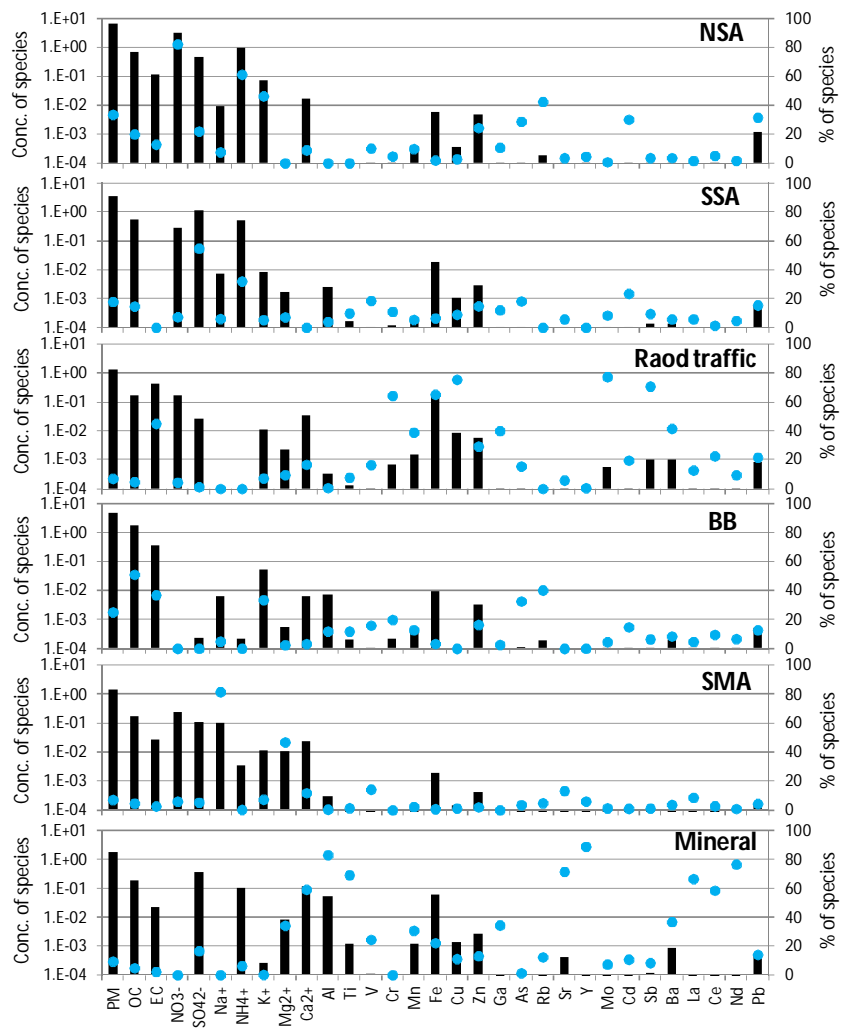

Figure S2: Chemical profiles of the sources detected at Zurich-Kaserne (ZUE; UB) and Payerne (PAY; RB).

- Mineral (MM), dominated by $\mathrm{Ca}^{2+}, \mathrm{Fe}, \mathrm{Al}$ and $\mathrm{Mg}^{2+}$, representing the main components of crustal matter. The mineral dust factors account moreover for a large mass fraction of crustal elements such as $\mathrm{Ti}, \mathrm{Sr}, \mathrm{Y}, \mathrm{La}, \mathrm{Ce}$ and $\mathrm{Nd}$;

- $\mathrm{Na}-\mathrm{Mg}$ rich (SS), contributing to high fractions of $\mathrm{Na}^{+}$and $\mathrm{Mg}^{2+}$. The contributions of the $\mathrm{Na}-\mathrm{Mg}$ rich factor did not show a clear annual cycle with elevated values during winter, thus suggesting a low contribution from the de- 
icing road salt. This source was mostly related to the transport of sea spray aerosol particles (Gianini et al., 2012).

- Secondary sulfate (SSA), characterized by high concentrations of $\mathrm{SO}_{4}{ }^{2-}$ and $\mathrm{NH}_{4}{ }^{+}$. Moreover, a relevant fraction of measured $\mathrm{OC}$ is also explained by the SSA factors; secondary $\mathrm{OC}$ is expected to be in receptor modelling studies largely associated with the secondary $\mathrm{SO}_{4}{ }^{2-}$ because of similar temporal variation of these constituents of atmospheric PM (Kim et al., 2003). Relatively high contents of $\mathrm{OC}$ in secondary sulfate factors have been attributed to the condensation of semi-volatile compounds on the high specific surface area of ammonium sulfate (Amato et al., 2009);

- Secondary nitrate (NSA), secondary inorganic source traced by $\mathrm{NO}_{3}{ }^{-}$and $\mathrm{NH}_{4}{ }^{+}$;

- Biomass burning $(B B)$, traced by high concentrations of $\mathrm{OC}, \mathrm{EC}$ and $\mathrm{K}^{+}$. This factor also explains a relevant mass fraction of $\mathrm{Rb}$, an element related to biomass combustion emissions (Godoy et al., 2005);

\section{- Source profiles from multi-site PMF for France.}

Figure S3 shows the chemical profiles of the sources from multi-site PMF for France. A total of 9 sources were identified at the French paired sites. A description of the sources is given below.

- Sea salt (SS), traced by $\mathrm{Na}^{+}$and $\mathrm{Cl}^{-}$this source represents mainly fresh marine aerosols;

- Land (or Primary) biogenic ( $L B$ ), traced by alcohols (arabitol and mannitol);

- Secondary sulfate (SSA), secondary inorganic aerosol traced by $\mathrm{SO}_{4}{ }^{2-}$ and $\mathrm{NH}_{4}^{+}$;

- Primary Road traffic (RT), traced by EC, OC, $\mathrm{Fe}, \mathrm{Cu}, \mathrm{Sb}$;

- Biomass burning (BB), traced mostly by levoglucosan and polysaccharides;

- Secondary nitrate (NSA), secondary inorganic aerosol traced by $\mathrm{NO}_{3}{ }^{-}$and $\mathrm{NH}_{4}{ }^{+}$;

- Marine aged, representing aged sea salt. Lack of $\mathrm{Cl}^{-}$in the chemical profiles and presence of $\mathrm{Na}^{+}$and $\mathrm{NO}_{3}{ }^{-}$;

- Mineral (MM), traced mainly by typical crustal elements such as $\mathrm{Fe}, \mathrm{Ca}, \mathrm{Al}, \mathrm{Sr}$ and $\mathrm{Ti}$;

- Marine biogenic $(M B)$, traced mainly by methane sulphonic acid, a product of DMS oxidation. 


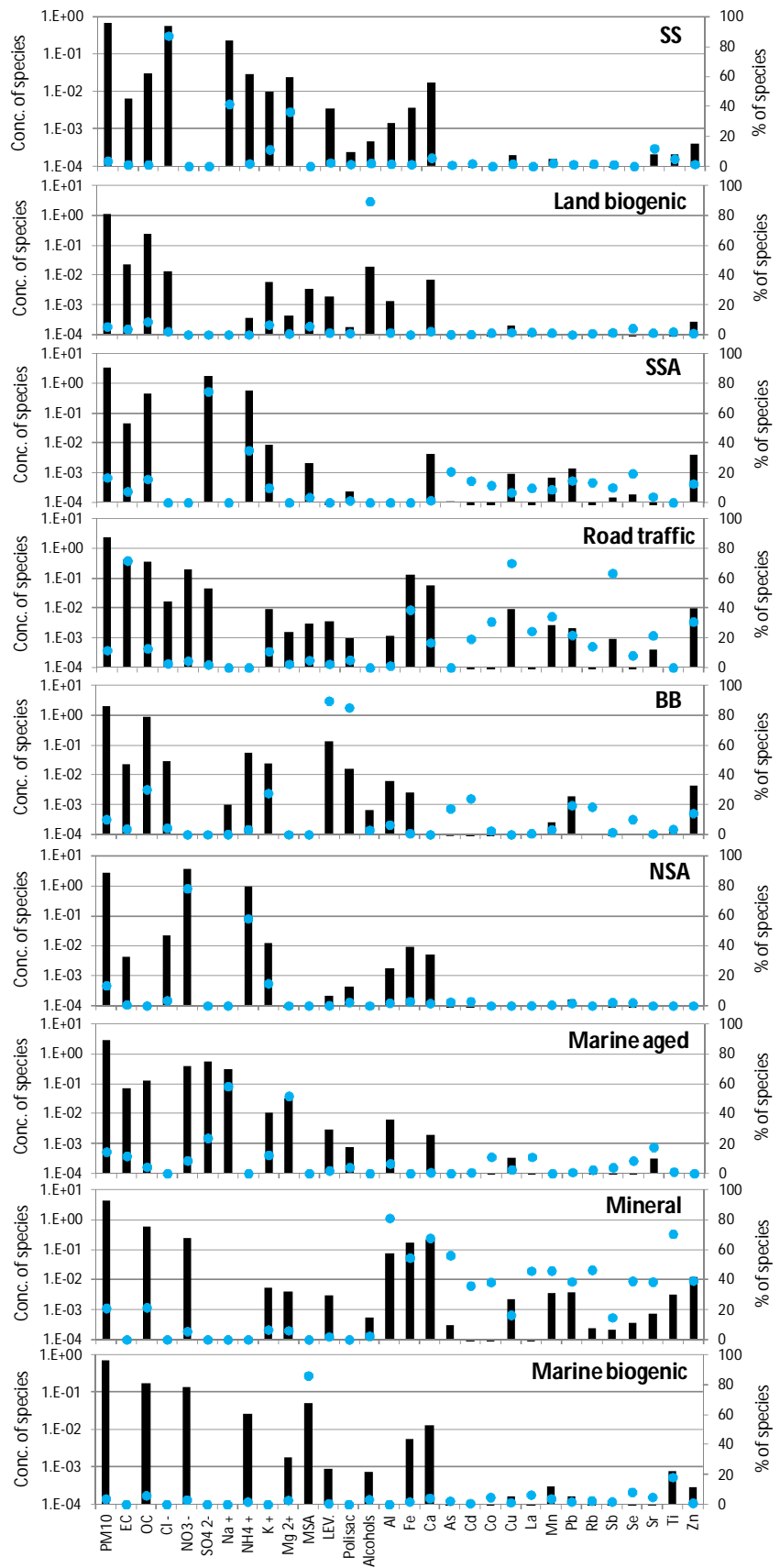

Figure S3: Chemical profiles of the sources detected at Lens (LENS; UB) and Revin (REV; RB). 


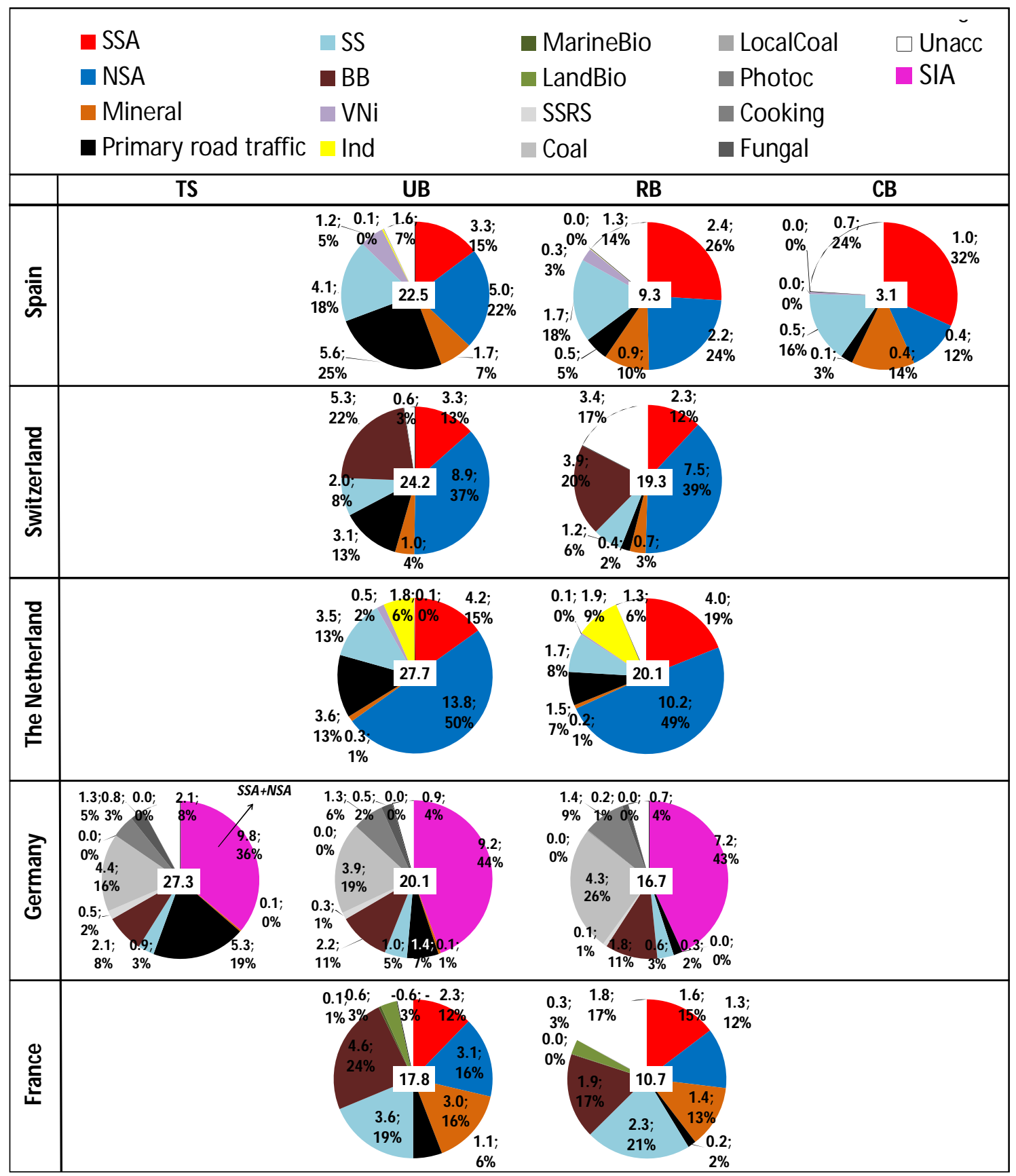

Figure S4: Source contributions to $\mathrm{PM}_{10}\left(\mathrm{PM}_{2.5}\right.$ for The Netherlands) in winter (DJF) from the multi-site PMF for each country. The number in the white box at the center of the pie chart is the measured mass of $\mathrm{PM}$ (in $\mu \mathrm{g} / \mathrm{m}^{3}$ ). TS: traffic site; UB: urban background; RB: regional background; $\mathrm{CB}$ : continental background. 


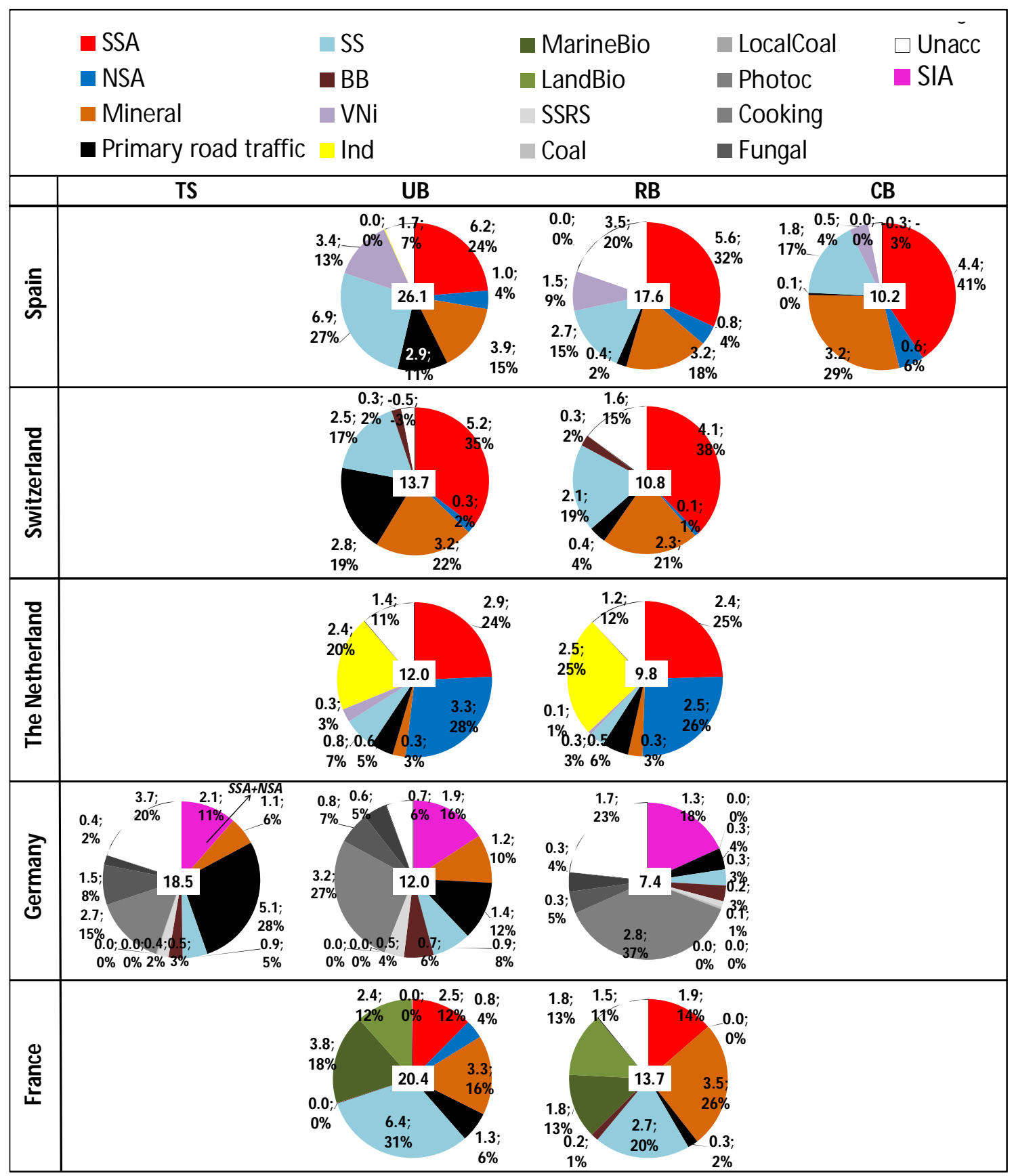

Figure S5: Source contributions to $\mathrm{PM}_{10}\left(\mathrm{PM}_{2.5}\right.$ for The Netherlands) in summer (JJA) from the multi-site PMF for each country. The number in the white box at the center of the pie chart is the measured mass of PM (in $\mu \mathrm{g} / \mathrm{m}^{3}$ ). TS: traffic site; UB: urban background; RB: regional background; CB: continental background. 


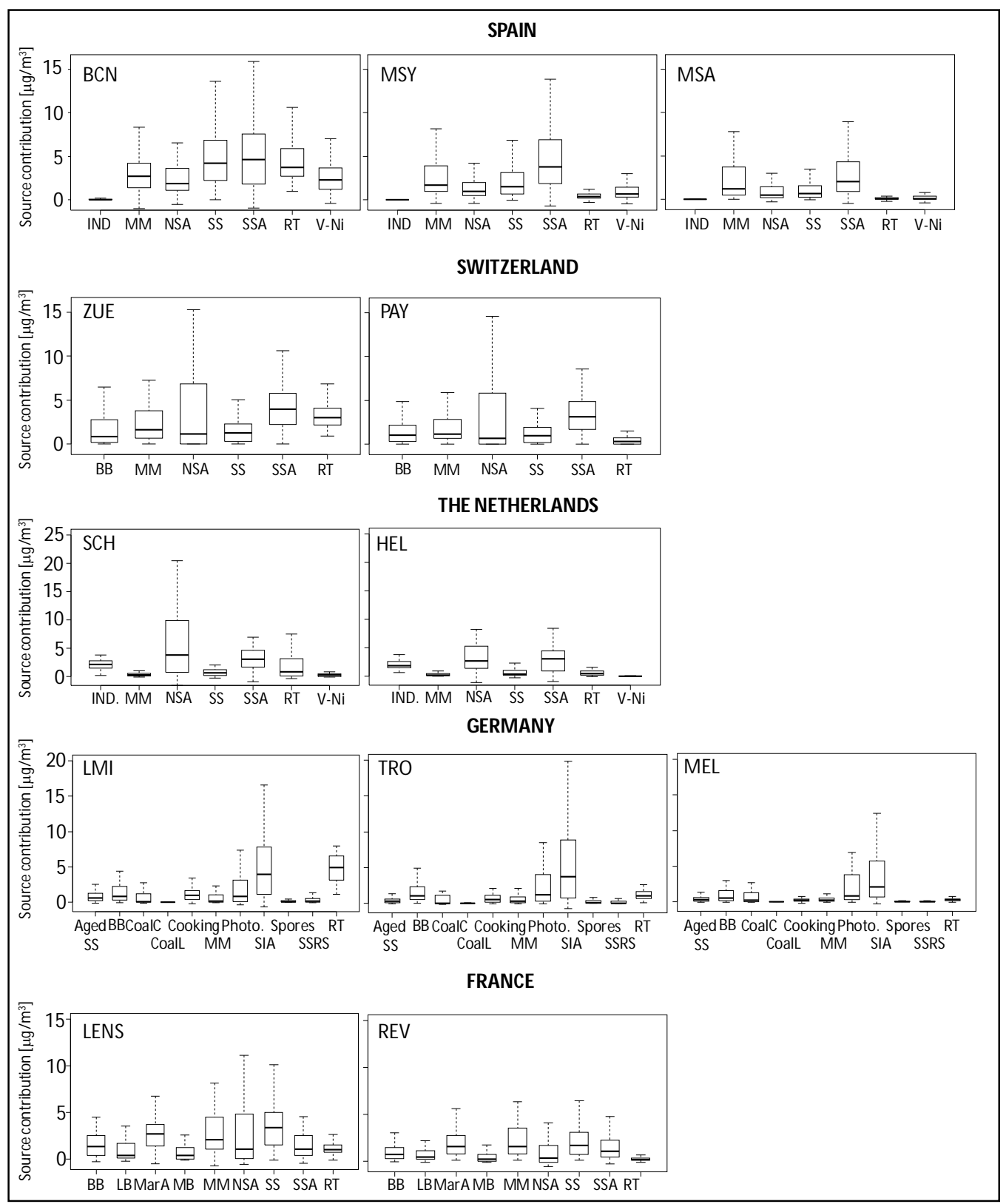

Figure S6: Mean annual source contributions to $\mathrm{PM}_{10}\left(\mathrm{PM}_{2.5}\right.$ for The Netherlands) from the multi-site PMF for each site and country. IND: Industrial; MM: Mineral matter; NSA: nitrate-rich particles; SS: Sea Salt; SSA: sulfate-rich particles; RT: Road traffic; V-Ni: Residual oil combustion; BB: Biomass burning; Photo: Photochemistry; CoalL: Coal local; SIA: Secondary inorganic aerosols; SSRS: Sea salt/Road dust; LB: Land biogenic; Mar A: Marine aged; MB: Marine biogenic. 


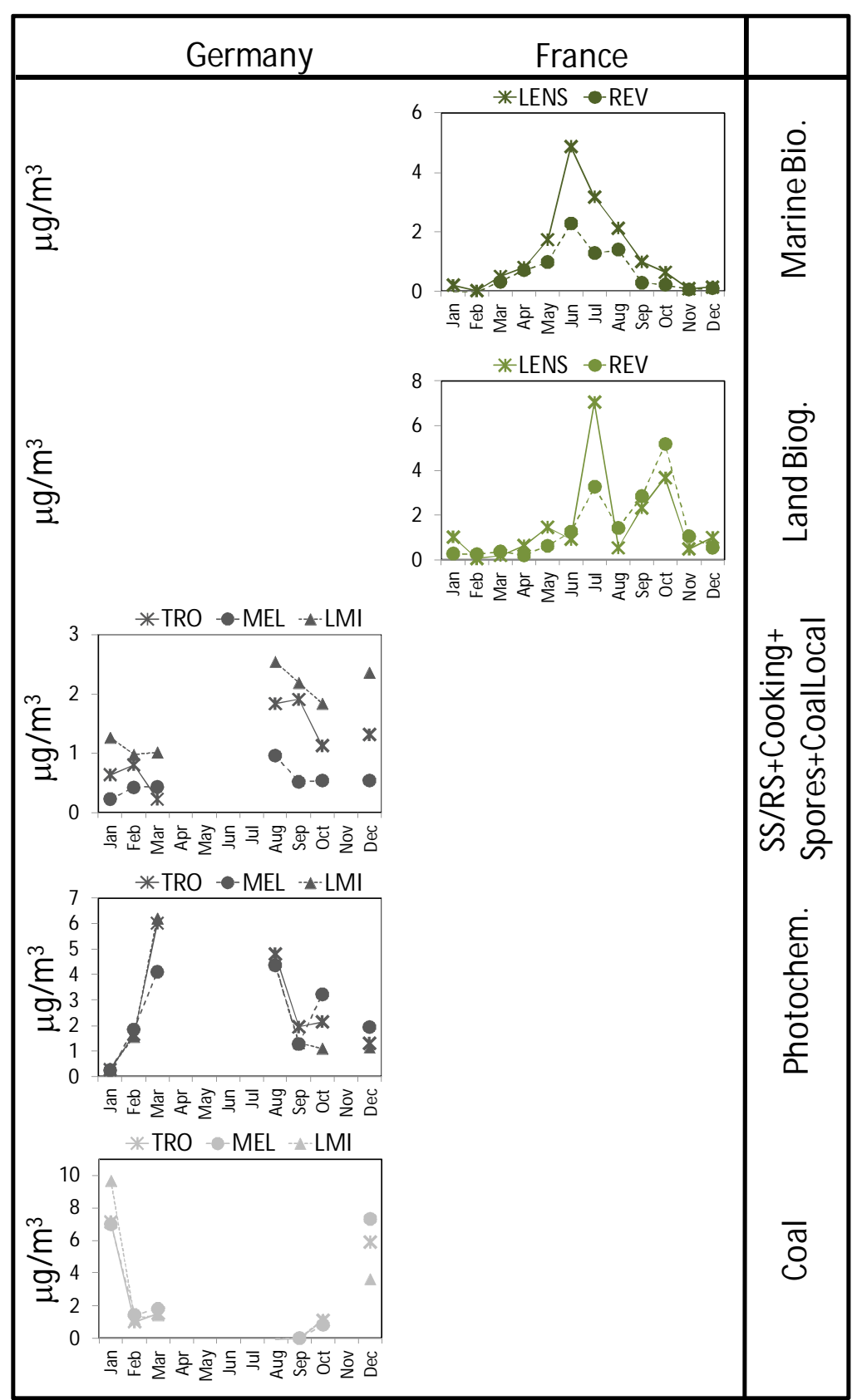

Figure S7: PMF source contributions for those sources only calculated at specific sites: France (Marine biogenic and Land biogenic); Germany (SS/RS+cooking+fungal spores+coal local, photochemistry, and coal). 
Concentration Weighted Trajectory (CWT) plat for Schiedam - $\mathrm{PM}_{2.5}$ NiN source (2007-2008)

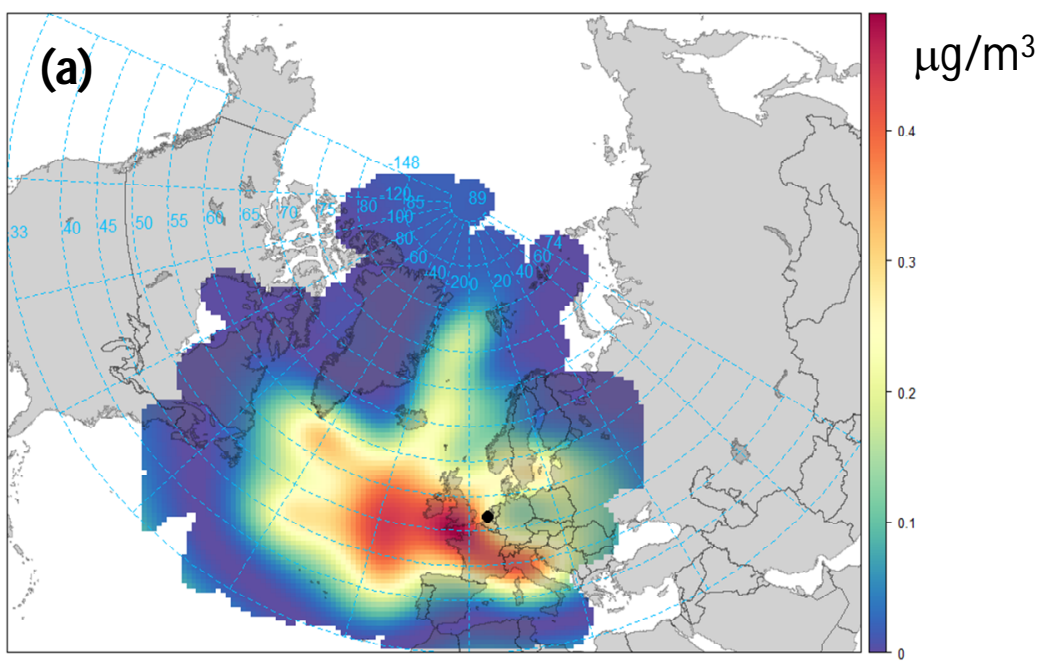

Concentration Weighted Trajectory (CWT) plot for Barcelona - $\mathrm{PM}_{10}$ NiN source (2010-2014)

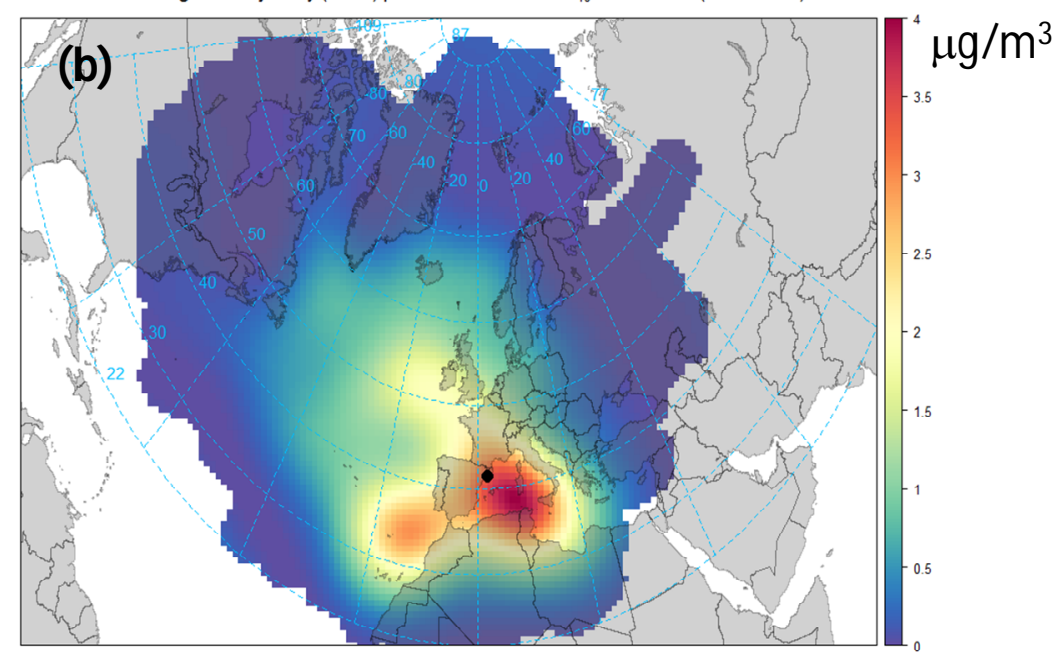

Figure S8: Concentration Weighted Trajectory (CWT) plots of the V-Ni source contributions for: (a) Schiedam (NL; $\mathrm{PM}_{2.5} ; 2007-2008$ ) and (b) Barcelona (ES; $\mathrm{PM}_{10} ; 2010-2014$ ). 


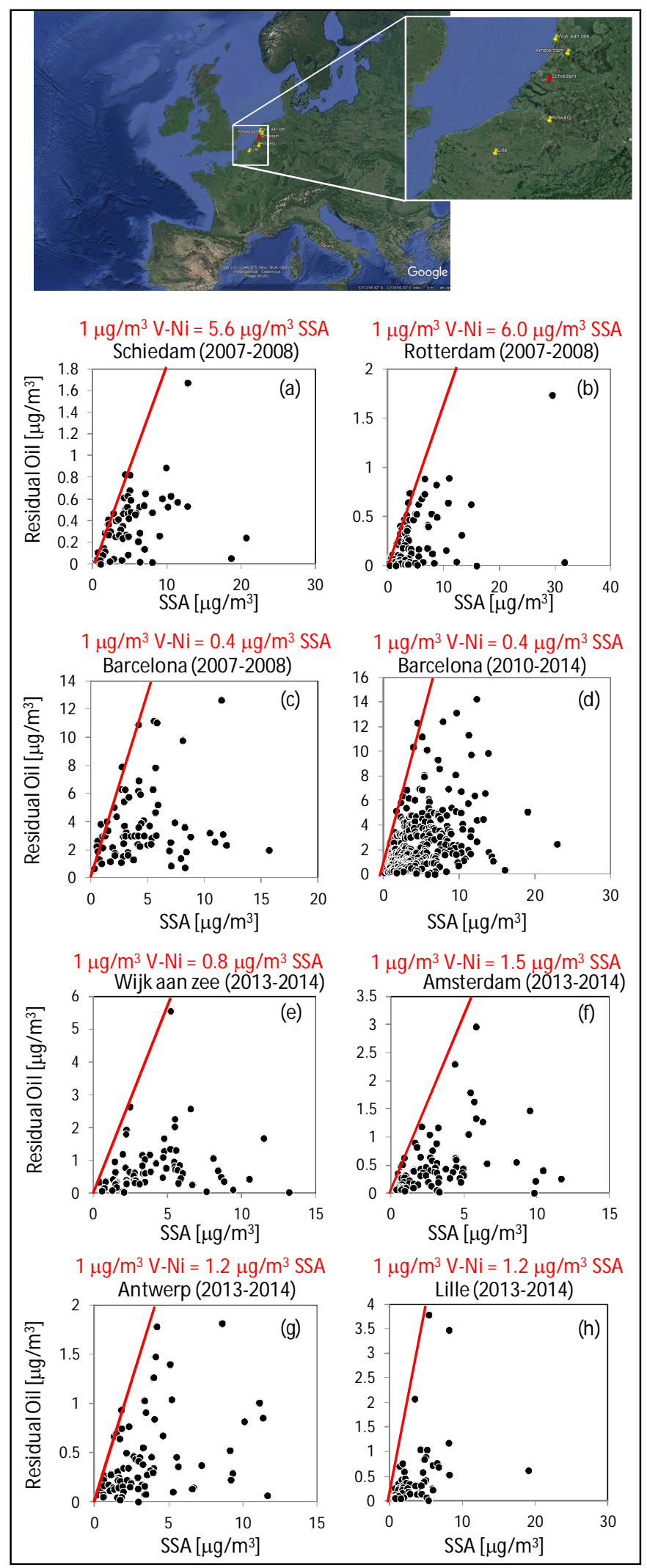

Figure S9: Contributions $\left(\mu \mathrm{g} / \mathrm{m}^{3}\right)$ of the V/Ni-bearing particles from shipping and SSA particles to PM at Schiedam (a), Rotterdam (b), Wijk aan zee (e), Amsterdam (f) in the Netherlands, Barcelona (c and d; Spain), Antwerp (g; Belgium) and Lille (h; France). The red lines represent the edges of the scatter plots. 

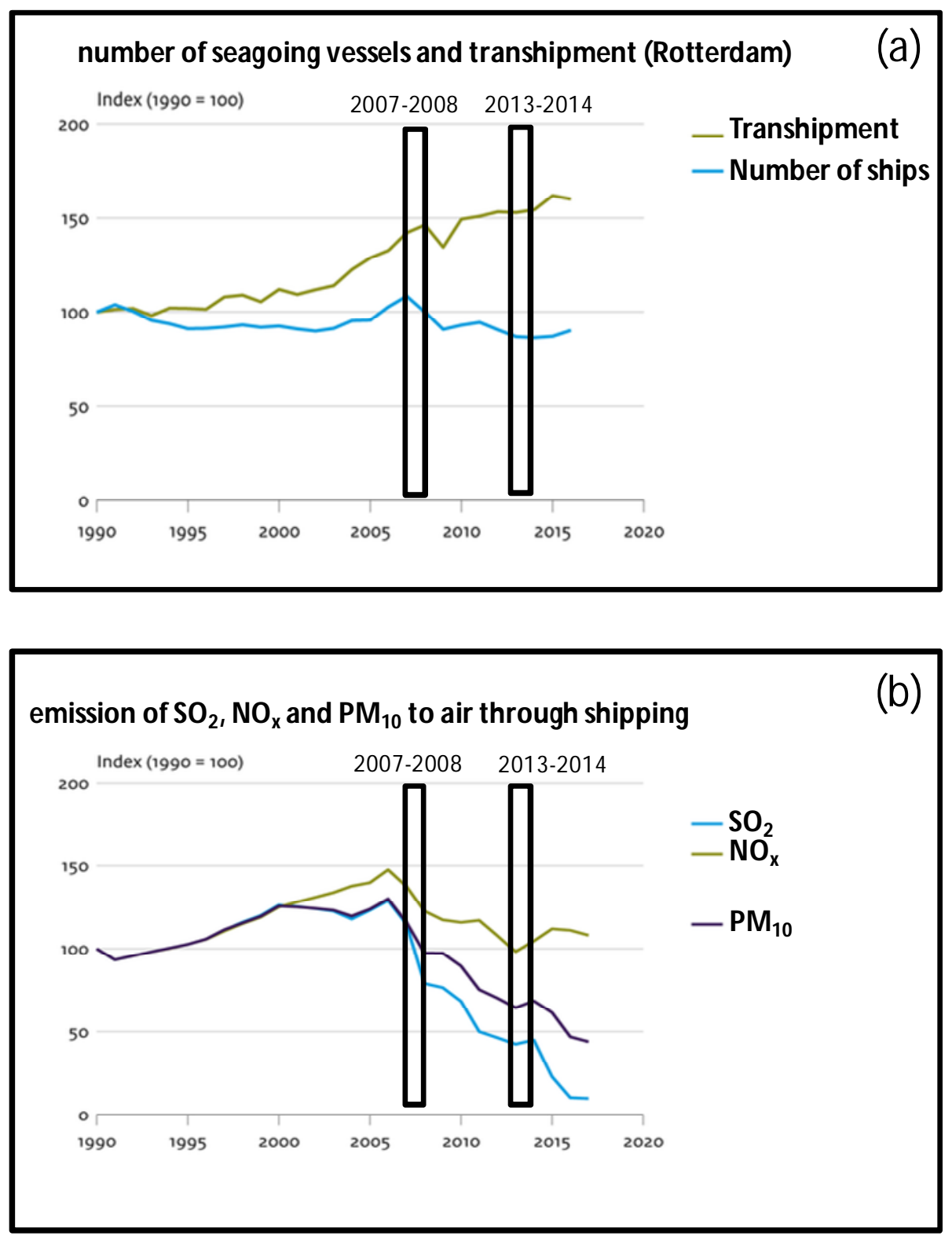

Figure S10: Number of seagoing vessels in Rotterdam (a), and emissions of $\mathrm{SO}_{2}, \mathrm{NO}_{\mathrm{x}}$ and $\mathrm{PM}_{10}$ through maritime shipping (b) from 1990 to 2017 (adapted from Environmental Data Compendium, Government of the Netherlands, https://www.clo.nl/en.) 


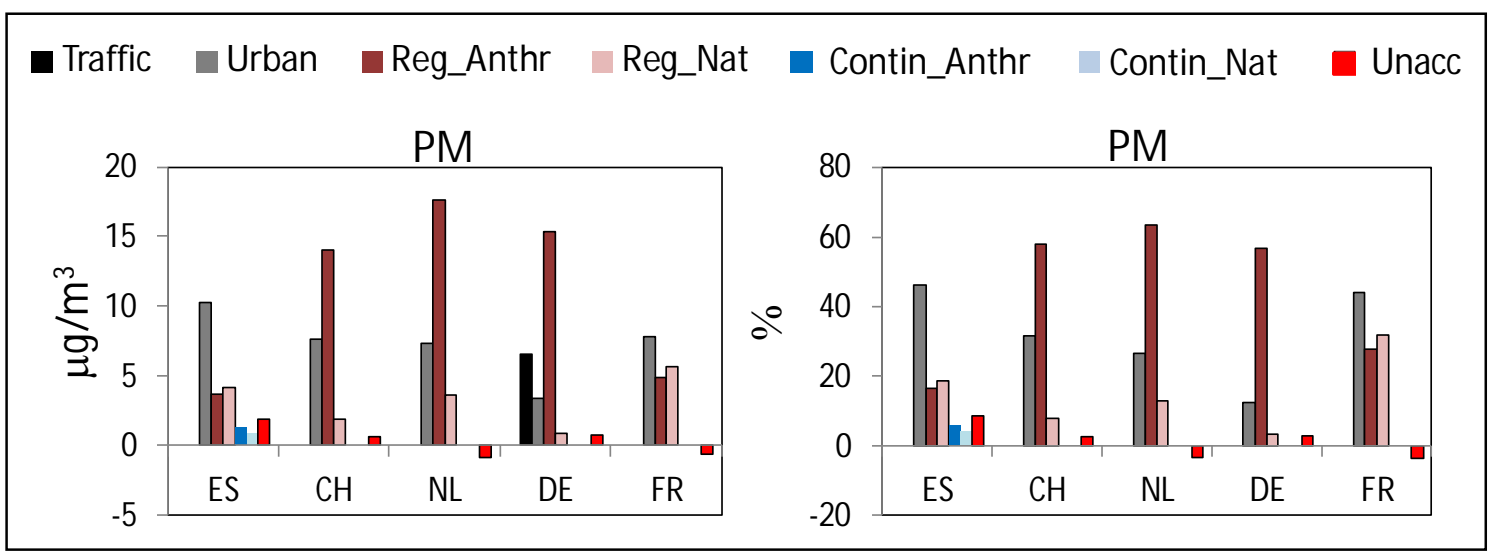

Figure S11: Lenschow's approach applied to the concentrations of PM. Average values for winter (DJF) are reported. ES: Spain; CH: Switzerland; NL: The Netherlands; DE: Germany; FR: France. In all countries with the exception of Spain, Reg_Anthr and Reg_Nat are the sum of regional+continental.

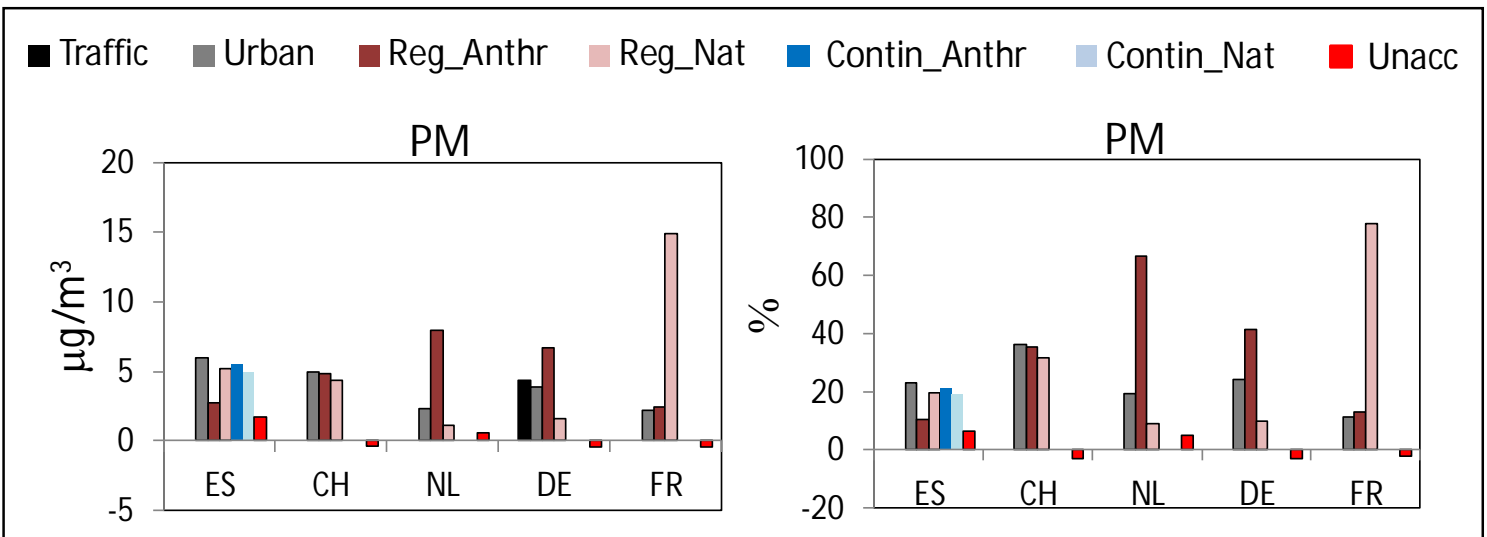

Figure S12: Lenschow's approach applied to the concentrations of PM. Average values for summer (JJA) are reported. ES: Spain; CH: Switzerland; NL: The Netherlands; DE: Germany; FR: France. In all countries with the exception of Spain, Reg_Anthr and Reg_Nat are the sum of regional+continental. 
-Traffic $\square$ Urban $\square$ Reg_Anthr $\square$ Reg_Nat $\square$ Contin_Anthr $\square$ Contin_Nat $\square$ Sum

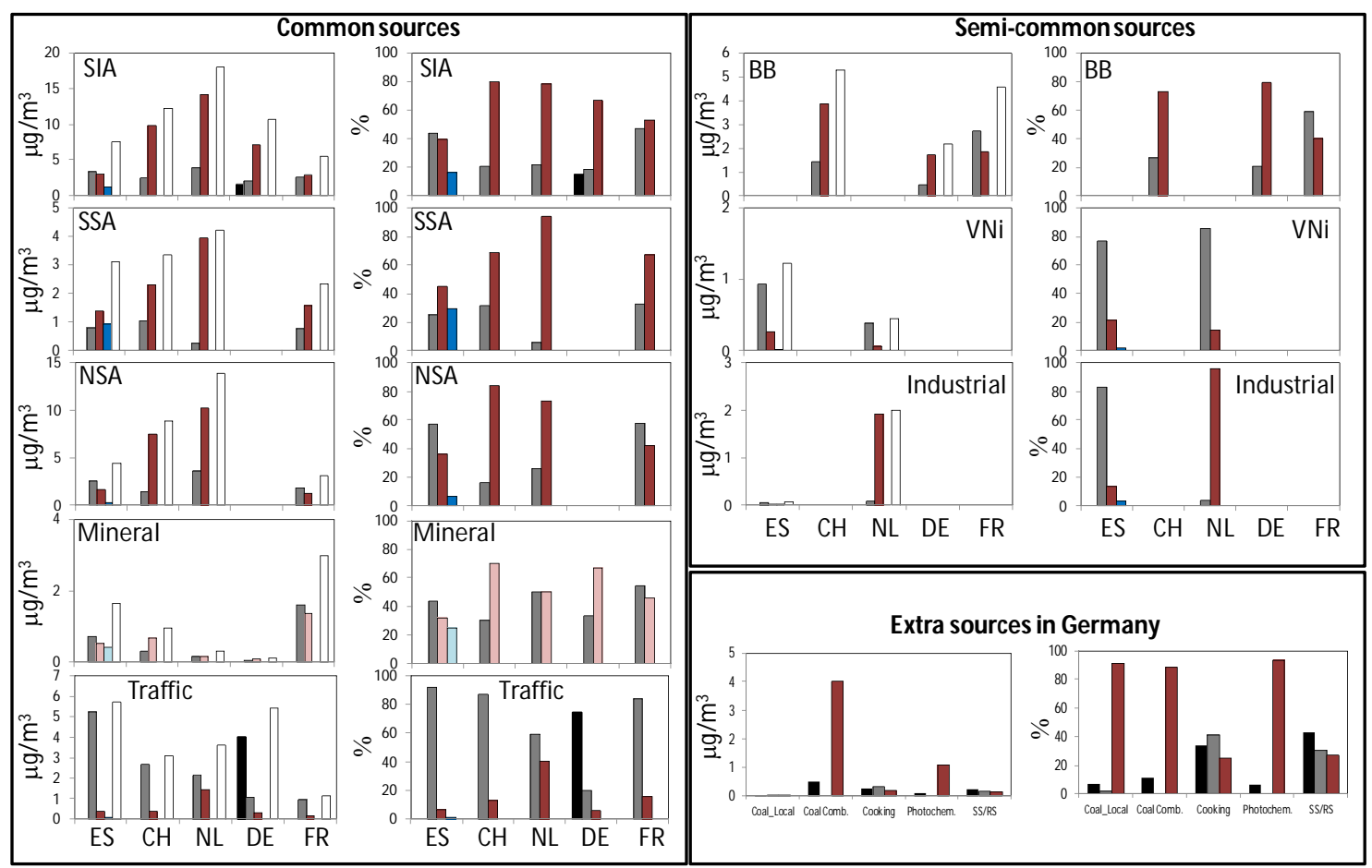

Figure S13: Lenschow's approach applied to the PM F source contributions. Average values for winter (DJF) are reported. ES: Spain; CH: Switzerland; NL: The Netherlands; DL: Germany; FR: France. In all countries with the exception of Spain, Reg_Anthr and Reg_Nat are the sum of regional+continental. 
-Traffic $\square$ Urban $\square$ Reg_Anthr $\square$ Reg_Nat $\square$ Contin_Anthr $\square$ Contin_Nat $\square$ Sum

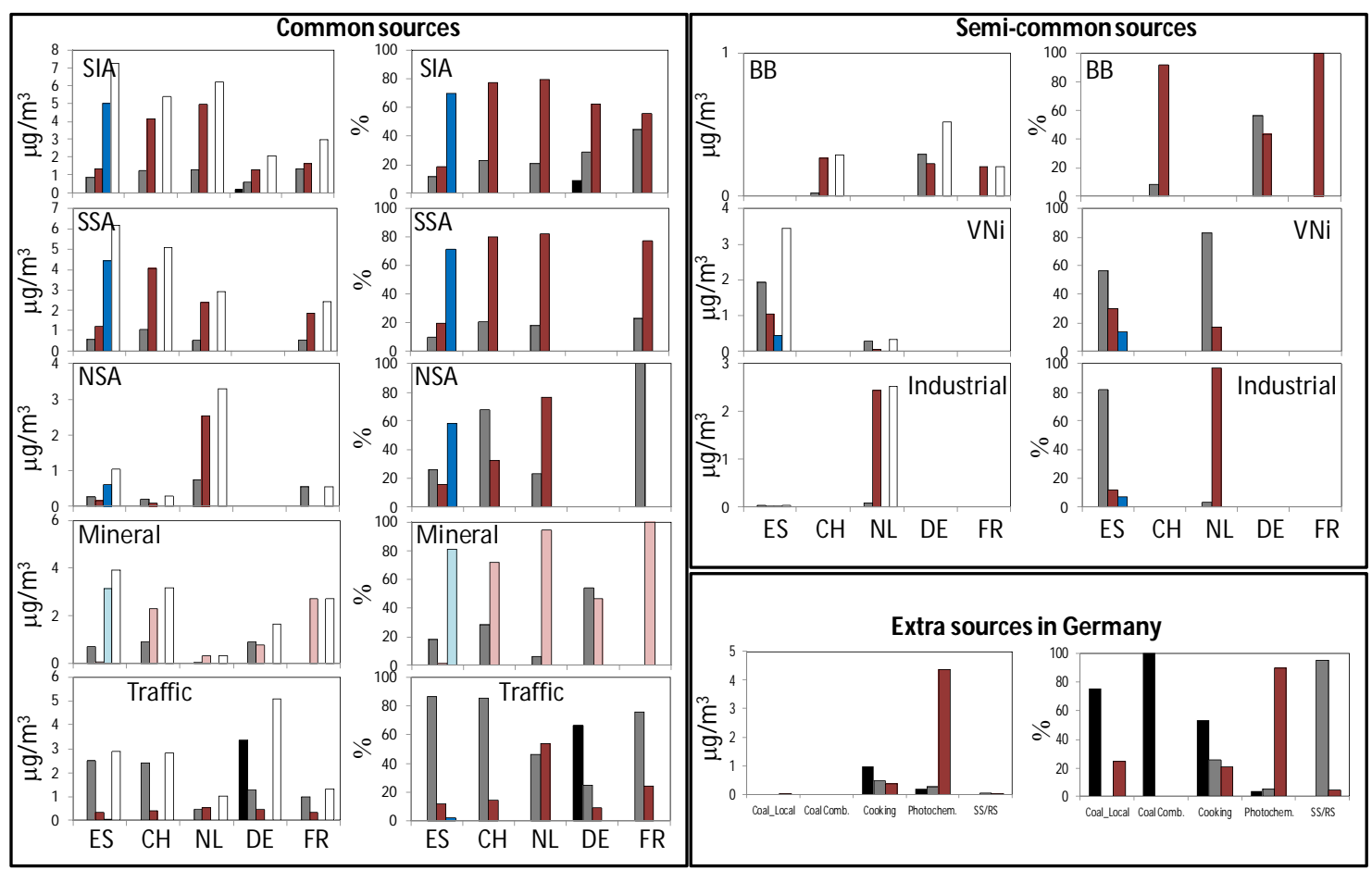

Figure S14: Lenschow's approach applied to the PM F source contributions. Average values for summer (JJA) are reported. ES: Spain; CH: Switzerland; NL: The Netherlands; DL: Germany; FR: France. In all countries, with the exception of Spain, Reg_Anthr and Reg_Nat are the sum of regional+continental. 
Table S5: Allocation of PM to different sources and origin in each country. Annual means and winter (DJF) and summer (JJA) averages are reported.

\begin{tabular}{|c|c|c|c|c|c|c|c|}
\hline & \multirow{3}{*}{$\begin{array}{l}\text { Contribution to } \mathrm{PM}^{(\mathrm{A})} \\
{\left[\mu \mathrm{g} / \mathrm{m}^{3} ; \% \text { of } \mathrm{PM} \text { mass }\right]}\end{array}$} & \multicolumn{6}{|c|}{ Annual mean } \\
\hline \multirow[t]{2}{*}{ Country } & & $\begin{array}{c}\text { Traffic } \\
{\left[\mu \mathrm{g} / \mathrm{m}^{3} ; \%\right]}\end{array}$ & $\begin{array}{c}\text { Urban } \\
{\left[\mu \mathrm{g} / \mathrm{m}^{3} ; \% \text { of }\right.} \\
\text { PM mass }]\end{array}$ & \multicolumn{2}{|c|}{$\begin{array}{c}\text { Regional } \\
{\left[\mu \mathrm{g} / \mathrm{m}^{3} ; \% \text { of } \mathrm{PM} \text { mass }\right]}\end{array}$} & \multicolumn{2}{|c|}{$\begin{array}{c}\text { Continental } \\
{\left[\mu \mathrm{g} / \mathrm{m}^{3} ; \% \text { of } \mathrm{PM}\right.} \\
\text { mass] }\end{array}$} \\
\hline & & Anthr. & Anthr. & Natural & Anthr. & Natural & Anthr. \\
\hline Spain & $24.4 ; 100$ & & $8.5 ; 35.0$ & $4.6 ; 18.8$ & $3.4 ; 13.8$ & $3.3 ; 13.5$ & $4.3 ; 17.8$ \\
\hline Switzerland & $19.3 ; 100$ & & $6.5 ; 33.7$ & $3.3 ; 17.0$ & $10.0 ; 51.9$ & & \\
\hline $\begin{array}{c}\text { The } \\
\text { Netherlands }\end{array}$ & $16.8 ; 100$ & & $4.3 ; 25.5$ & $2.0 ; 12.1$ & $10.5 ; 62.1$ & & \\
\hline Germany & $21.6 ; 100$ & $5.4 ; 24.8$ & $3.5 ; 16.3$ & $1.1 ; 5.0$ & $10.8 ; 50.0$ & & \\
\hline \multirow[t]{4}{*}{ France } & $20.7 ; 100$ & & $6.7 ; 32.6$ & $9.1 ; 43.9$ & $4.6 ; 22.5$ & & \\
\hline & & \multicolumn{6}{|c|}{ Winter } \\
\hline & $\begin{array}{c}\text { Contribution to PM } \\
{\left[\mu \mathrm{g} / \mathrm{m}^{3} ; \% \text { of } \mathrm{PM} \text { mass] }\right.}\end{array}$ & $\begin{array}{c}\text { Traffic } \\
{\left[\mu \mathrm{g} / \mathrm{m}^{3} ; \%\right]}\end{array}$ & $\begin{array}{c}\text { Urban } \\
{\left[\mu \mathrm{g} / \mathrm{m}^{3} ; \% \text { of }\right.} \\
\text { PM mass] }\end{array}$ & \multicolumn{2}{|c|}{$\begin{array}{c}\text { Regional } \\
{\left[\mu \mathrm{g} / \mathrm{m}^{3} ; \% \text { of PM mass }\right]}\end{array}$} & \multicolumn{2}{|c|}{$\begin{array}{c}\text { Continental } \\
{\left[\mu \mathrm{g} / \mathrm{m}^{3} ; \% \text { of } \mathrm{PM}\right.} \\
\text { mass }]\end{array}$} \\
\hline & & Anthr. & Anthr. & Natural & Anthr. & Natural & Anthr. \\
\hline Spain & $22.3 ; 100$ & & $10.3 ; 46.1$ & $4.2 ; 18.8$ & $3.7 ; 16.5$ & $0.9 ; 4.0$ & $1.3 ; 6.0$ \\
\hline Switzerland & $24.2 ; 100$ & & $7.6 ; 31.5$ & $1.9 ; 7.9$ & $14.0 ; 58.0$ & & \\
\hline $\begin{array}{c}\text { The } \\
\text { Netherlands }\end{array}$ & $27.7 ; 100$ & & $7.4 ; 26.6$ & $3.6 ; 13.1$ & $17.6 ; 63.5$ & & \\
\hline Germany & $27.0 ; 100$ & $6.6 ; 24.3$ & $3.4 ; 12.5$ & $0.9 ; 3.4$ & $15.4 ; 56.9$ & & \\
\hline \multirow[t]{4}{*}{ France } & $17.8 ; 100$ & & $7.8 ; 44.0$ & $5.7 ; 31.9$ & $4.9 ; 27.6$ & & \\
\hline & & \multicolumn{6}{|c|}{ Summer } \\
\hline & $\begin{array}{c}\text { Contribution to PM } \\
{\left[\mu \mathrm{g} / \mathrm{m}^{3} ; \% \text { of } \mathrm{PM} \text { mass] }\right.}\end{array}$ & $\begin{array}{c}\text { Traffic } \\
{\left[\mu \mathrm{g} / \mathrm{m}^{3} ; \%\right]}\end{array}$ & $\begin{array}{c}\text { Urban } \\
{\left[\mu \mathrm{g} / \mathrm{m}^{3} ; \% \text { of }\right.} \\
\text { PM mass }]\end{array}$ & \multicolumn{2}{|c|}{$\begin{array}{c}\text { Regional } \\
{\left[\mu \mathrm{g} / \mathrm{m}^{3} ; \% \text { of PM mass }\right]}\end{array}$} & \multicolumn{2}{|c|}{$\begin{array}{c}\text { Continental } \\
{\left[\mu \mathrm{g} / \mathrm{m}^{3} ; \% \text { of } \mathrm{PM}\right.} \\
\text { mass }]\end{array}$} \\
\hline & & Anthr. & Anthr. & Natural & Anthr. & Natural & Anthr. \\
\hline Spain & $26.1 ; 100$ & & $6.0 ; 23.1$ & $5.2 ; 19.7$ & $2.7 ; 10.4$ & $5.0 ; 19.1$ & $5.5 ; 21.2$ \\
\hline Switzerland & $13.7 ; 100$ & & $5.0 ; 36.3$ & $4.3 ; 31.7$ & $4.8 ; 35.1$ & & \\
\hline $\begin{array}{c}\text { The } \\
\text { Netherlands }\end{array}$ & $12.0 ; 100$ & & $2.3 ; 19.3$ & $1.1 ; 9.2$ & $8.0 ; 66.6$ & & \\
\hline Germany & $16.0 ; 100$ & $4.3 ; 27.3$ & $3.9 ; 24.2$ & $1.6 ; 9.8$ & $6.6 ; 41.6$ & & \\
\hline France & $19.1 ; 100$ & & $2.2 ; 11.5$ & $14.9 ; 77.9$ & $2.5 ; 12.9$ & & \\
\hline
\end{tabular}

(A) PM concentrations measured in Barcelona (BCN; Spain), Zurich (ZUE; Switzerland), Schiedam (SCH; The Netherlands), Leipzig-Mitte (LMI; Germany) and Lens (LENS; France). 
Table S6: Allocation of PMF source contributions in each country. Annual means are reported.

\begin{tabular}{|c|c|c|c|c|c|c|c|}
\hline & \multirow{3}{*}{$\begin{array}{c}\text { Source contribution }^{(A)} \\
\text { SIA } \\
{\left[\mu \mathrm{g} / \mathrm{m}^{3} ; \% \text { of PM mass }\right]}\end{array}$} & \multicolumn{6}{|c|}{ Annual mean } \\
\hline & & $\begin{array}{c}\text { Traffic } \\
{\left[\mu \mathrm{g} / \mathrm{m}^{3} ; \% \text { of } \mathrm{SIA}\right]}\end{array}$ & $\begin{array}{c}\text { Urban } \\
{\left[\mu \mathrm{g} / \mathrm{m}^{3} ; \% \text { of }\right.} \\
\mathrm{SIA}]\end{array}$ & \multicolumn{2}{|c|}{$\begin{array}{c}\text { Regional } \\
{\left[\mu \mathrm{g} / \mathrm{m}^{3} ; \% \text { of } \mathrm{SIA}\right]}\end{array}$} & \multicolumn{2}{|c|}{$\begin{array}{c}\text { Continental } \\
{\left[\mu \mathrm{g} / \mathrm{m}^{3} ; \% \text { of } \mathrm{SIA}\right]}\end{array}$} \\
\hline & & Anthr. & Anthr. & Natural & Anthr. & Natural & Anthr. \\
\hline Spain & $8.2 ; 33.6$ & & $1.9 ; 23.8$ & & $2.2 ; 27.4$ & & $4.0 ; 48.8$ \\
\hline Switzerland & $9.1 ; 46.9$ & & $1.7 ; 18.6$ & & $7.7 ; 85.4$ & & \\
\hline $\begin{array}{c}\text { The } \\
\text { Netherlands }\end{array}$ & $9.8 ; 58.2$ & & $2.1 ; 21.0$ & & $7.7 ; 79.0$ & & \\
\hline Germany & $6.2 ; 26.9$ & $0.8 ; 13.5$ & $1.3 ; 20.6$ & & $4.4 ; 71.7$ & & \\
\hline \multirow[t]{4}{*}{ France } & $5.8 ; 28.2$ & & $2.5 ; 43.5$ & & $3.3 ; 56.5$ & & \\
\hline & & \multicolumn{6}{|c|}{ Annual mean } \\
\hline & $\begin{array}{c}\text { SSA } \\
{\left[\mu \mathrm{g} / \mathrm{m}^{3} ; \% \text { of PM mass }\right]}\end{array}$ & $\begin{array}{c}\text { Traffic } \\
{\left[\mu \mathrm{g} / \mathrm{m}^{3} ; \% \text { of }\right.} \\
\mathrm{SSA}]\end{array}$ & $\begin{array}{c}\text { Urban } \\
{\left[\mu \mathrm{g} / \mathrm{m}^{3} ; \% \text { of }\right.} \\
\mathrm{SSA}]\end{array}$ & \multicolumn{2}{|c|}{$\begin{array}{c}\text { Regional } \\
{\left[\mu \mathrm{g} / \mathrm{m}^{3} ; \% \text { of } \mathrm{SSA}\right]}\end{array}$} & \multicolumn{2}{|c|}{$\begin{array}{c}\text { Continental } \\
{\left[\mu \mathrm{g} / \mathrm{m}^{3} ; \% \text { of } \mathrm{SSA}\right]}\end{array}$} \\
\hline & & Anthr. & Anthr. & Natural & Anthr. & Natural & Anthr. \\
\hline Spain & $5.2 ; 21.4$ & & $0.5 ; 8.7$ & & $1.8 ; 33.8$ & & $3.0 ; 57.5$ \\
\hline Switzerland & $4.6 ; 23.7$ & & $1.1 ; 23.7$ & & $3.5 ; 76.8$ & & \\
\hline $\begin{array}{c}\text { The } \\
\text { Netherlands }\end{array}$ & $3.6 ; 21.2$ & & $0.7 ; 18.8$ & & $2.9 ; 81.2$ & & \\
\hline \multicolumn{8}{|l|}{ Germany } \\
\hline \multirow[t]{4}{*}{ France } & $2.2 ; 10.6$ & & $0.4 ; 17.4$ & & $1.8 ; 82.6$ & & \\
\hline & & \multicolumn{6}{|c|}{ Annual mean } \\
\hline & $\begin{array}{c}\text { NSA } \\
{\left[\mu \mathrm{g} / \mathrm{m}^{3} ; \% \text { of PM mass }\right]}\end{array}$ & $\begin{array}{c}\text { Traffic } \\
{\left[\mu \mathrm{g} / \mathrm{m}^{3} ; \% \text { of }\right.} \\
\mathrm{NSA}]\end{array}$ & $\begin{array}{c}\text { Urban } \\
{\left[\mu \mathrm{g} / \mathrm{m}^{3} ; \% \text { of }\right.} \\
\mathrm{NSA}]\end{array}$ & \multicolumn{2}{|c|}{$\begin{array}{c}\text { Regional } \\
{\left[\mu \mathrm{g} / \mathrm{m}^{3} ; \% \text { of } \mathrm{NSA}\right]}\end{array}$} & \multicolumn{2}{|c|}{$\begin{array}{c}\text { Continental } \\
{\left[\mu \mathrm{g} / \mathrm{m}^{3} ; \% \text { of } \mathrm{NSA}\right]}\end{array}$} \\
\hline & & Anthr. & Anthr. & Natural & Anthr. & Natural & Anthr. \\
\hline Spain & $3.0 ; 12.2$ & & $1.5 ; 50.3$ & & $0.5 ; 16.1$ & & $1.0 ; 33.6$ \\
\hline Switzerland & $4.5 ; 23.2$ & & $0.6 ; 13.5$ & & $4.2 ; 94.2$ & & \\
\hline $\begin{array}{c}\text { The } \\
\text { Netherlands }\end{array}$ & $6.2 ; 36.9$ & & $1.4 ; 22.2$ & & $4.8 ; 77.8$ & & \\
\hline \multicolumn{8}{|l|}{ Germany } \\
\hline \multirow[t]{4}{*}{ France } & $3.6 ; 17.5$ & & $2.1 ; 59.3$ & & $1.5 ; 40.7$ & & \\
\hline & & \multicolumn{6}{|c|}{ Annual mean } \\
\hline & $\begin{array}{c}\text { Mineral } \\
{\left[\mu \mathrm{g} / \mathrm{m}^{3} ; \% \text { of PM mass }\right]}\end{array}$ & $\begin{array}{c}\text { Traffic } \\
{\left[\mu \mathrm{g} / \mathrm{m}^{3} ; \% \text { of }\right.} \\
\text { Mineral] }\end{array}$ & $\begin{array}{c}\text { Urban } \\
{\left[\mu \mathrm{g} / \mathrm{m}^{3} ; \% \text { of }\right.} \\
\text { Mineral] }\end{array}$ & \multicolumn{2}{|c|}{$\begin{array}{c}\text { Regional } \\
{\left[\mu \mathrm{g} / \mathrm{m}^{3} ; \% \text { of Mineral }\right]}\end{array}$} & \multicolumn{2}{|c|}{$\begin{array}{c}\text { Continental } \\
{\left[\mu \mathrm{g} / \mathrm{m}^{3} ; \% \text { of Mineral }\right]}\end{array}$} \\
\hline & & Anthr. & Anthr. & Natural & Anthr. & Natural & Anthr. \\
\hline Spain & $3.3 ; 13.6$ & & $0.7 ; 20.5$ & $0.4 ; 13.2$ & & $2.2 ; 66.3$ & \\
\hline Switzerland & $2.6 ; 13.4$ & & $0.9 ; 33.1$ & $1.9 ; 73.7$ & & & \\
\hline $\begin{array}{c}\text { The } \\
\text { Netherlands }\end{array}$ & $0.5 ; 3.2$ & & $0.1 ; 27.5$ & $0.4 ; 72.5$ & & & \\
\hline Germany & $0.6 ; 2.4$ & $0.0 ; 0.0$ & $0.4 ; 70.4$ & $0.3 ; 57.7$ & & & \\
\hline \multirow[t]{4}{*}{ France } & $5.0 ; 24.3$ & & $1.8 ; 35.3$ & $3.2 ; 64.7$ & & & \\
\hline & & \multicolumn{6}{|c|}{ Annual mean } \\
\hline & $\begin{array}{c}\text { Road traffic } \\
{\left[\mu \mathrm{g} / \mathrm{m}^{3} ; \% \text { of } \mathrm{PM} \text { mass }\right]}\end{array}$ & $\begin{array}{c}\text { Traffic } \\
{\left[\mu \mathrm{g} / \mathrm{m}^{3} ; \% \text { of } \mathrm{RT}\right]}\end{array}$ & $\begin{array}{c}\text { Urban } \\
{\left[\mu \mathrm{g} / \mathrm{m}^{3} ; \% \text { of }\right.} \\
\mathrm{RT}]\end{array}$ & \multicolumn{2}{|c|}{$\begin{array}{c}\text { Regional } \\
{\left[\mu \mathrm{g} / \mathrm{m}^{3} ; \% \text { of } \mathrm{RT}\right]}\end{array}$} & \multicolumn{2}{|c|}{$\begin{array}{c}\text { Continental } \\
{\left[\mu \mathrm{g} / \mathrm{m}^{3} ; \% \text { of } \mathrm{RT}\right]}\end{array}$} \\
\hline & & Anthr. & Anthr. & Natural & Anthr. & Natural & Anthr. \\
\hline Spain & $4.7 ; 19.1$ & & $4.2 ; 90.0$ & & $0.4 ; 8.1$ & & $0.1 ; 1.9$ \\
\hline Switzerland & $3.6 ; 18.5$ & & $3.0 ; 84.3$ & & $0.5 ; 13.4$ & & \\
\hline $\begin{array}{c}\text { The } \\
\text { Netherlands }\end{array}$ & $2.0 ; 11.9$ & & $1.2 ; 62.2$ & & $0.7 ; 36.1$ & & \\
\hline Germany & $5.2 ; 22.6$ & $3.8 ; 73.0$ & $1.1 ; 20.9$ & & $0.3 ; 6.1$ & & \\
\hline France & $1.2 ; 5.6$ & & $0.9 ; 79.2$ & & $0.2 ; 20.8$ & & \\
\hline & & & & nnual me & & & \\
\hline & $\begin{array}{c}\text { SS } \\
{\left[\mu \mathrm{g} / \mathrm{m}^{3} ; \% \text { of } \mathrm{PM} \text { mass }\right]}\end{array}$ & $\begin{array}{c}\text { Traffic } \\
{\left[\mu \mathrm{g} / \mathrm{m}^{3} ; \% \text { of } \mathrm{SS}\right]}\end{array}$ & $\begin{array}{c}\text { Urban } \\
{\left[\mu \mathrm{g} / \mathrm{m}^{3} ; \% \text { of }\right.} \\
\mathrm{SS}]\end{array}$ & $\begin{array}{r}R € \\
{\left[\mu \mathrm{g} / \mathrm{m}^{3}\right.}\end{array}$ & of SS] & $\begin{array}{r}\text { Con } \\
{\left[\mu \mathrm{g} / \mathrm{m}^{3}\right.}\end{array}$ & $\begin{array}{l}\text { lental } \\
\% \text { of SS] }\end{array}$ \\
\hline & & Anthr. & Anthr. & Natural & Anthr. & Natural & Anthr. \\
\hline Spain & $5.2 ; 21.5$ & & & $5.2 ; 100$ & & & \\
\hline Switzerland & $1.7 ; 9.0$ & & & $1.7 ; 100$ & & & \\
\hline
\end{tabular}




\begin{tabular}{|c|c|c|c|c|c|c|c|}
\hline $\begin{array}{c}\text { The } \\
\text { Netherlands }\end{array}$ & $1.6 ; 9.7$ & & & $1.6 ; 100$ & & & \\
\hline Germany & $0.9 ; 4.0$ & & & $0.9 ; 100$ & & & \\
\hline \multirow[t]{4}{*}{ France } & $3.7 ; 17.7$ & & & $3.7 ; 100$ & & & \\
\hline & & \multicolumn{6}{|c|}{ Annual mean } \\
\hline & $\begin{array}{l}\text { Biomass burning } \\
{\left[\mu \mathrm{g} / \mathrm{m}^{3} ; \% \text { of } \mathrm{PM} \text { mass] }\right.}\end{array}$ & $\begin{array}{c}\text { Traffic } \\
{\left[\mu \mathrm{g} / \mathrm{m}^{3} ; \% \text { of } \mathrm{BB}\right]}\end{array}$ & $\begin{array}{c}\text { Urban } \\
{\left[\mu \mathrm{g} / \mathrm{m}^{3} ; \% \text { of }\right.} \\
\mathrm{BB}]\end{array}$ & \multicolumn{2}{|c|}{$\begin{array}{c}\text { Regional } \\
{\left[\mu \mathrm{g} / \mathrm{m}^{3} ; \% \text { of } \mathrm{BB}\right]}\end{array}$} & \multicolumn{2}{|c|}{$\begin{array}{c}\text { Continental } \\
{\left[\mu \mathrm{g} / \mathrm{m}^{3} ; \% \text { of } \mathrm{BB}\right]}\end{array}$} \\
\hline & & Anthr. & Anthr. & Natural & Anthr. & Natural & Anthr. \\
\hline \multicolumn{8}{|l|}{ Spain } \\
\hline Switzerland & $2.3 ; 12.0$ & & $0.6 ; 25.4$ & & $1.8 ; 78.1$ & & \\
\hline \multicolumn{8}{|l|}{$\begin{array}{c}\text { The } \\
\text { Netherlands }\end{array}$} \\
\hline Germany & $1.4 ; 6.0$ & $0.0 ; 0.0$ & $0.3 ; 23.2$ & & $1.1 ; 76.9$ & & \\
\hline \multirow[t]{4}{*}{ France } & $2.6 ; 12.8$ & & $1.5 ; 57.6$ & & $1.1 ; 42.4$ & & \\
\hline & & \multicolumn{6}{|c|}{ Annual mean } \\
\hline & $\begin{array}{l}\text { V-Ni } \\
\qquad\left[\mu \mathrm{g} / \mathrm{m}^{3} ; \% \text { of PM mass] }\right.\end{array}$ & $\begin{array}{c}\text { Traffic } \\
{\left[\mu \mathrm{g} / \mathrm{m}^{3} ; \% \text { of } \mathrm{V}-\right.} \\
\mathrm{Ni}]\end{array}$ & $\begin{array}{c}\text { Urban } \\
{\left[\mu \mathrm{g} / \mathrm{m}^{3} ; \% \text { of }\right.} \\
\text { V-Ni] }\end{array}$ & \multicolumn{2}{|c|}{$\begin{array}{c}\text { Regional } \\
{\left[\mu \mathrm{g} / \mathrm{m}^{3} ; \% \text { of } \mathrm{V}-\mathrm{Ni}\right]}\end{array}$} & \multicolumn{2}{|c|}{$\begin{array}{c}\text { Continental } \\
{\left[\mu \mathrm{g} / \mathrm{m}^{3} ; \% \text { of } \mathrm{V}-\mathrm{Ni}\right]}\end{array}$} \\
\hline & & Anthr. & Anthr. & Natural & Anthr. & Natural & Anthr. \\
\hline Spain & $2.7 ; 10.9$ & & $1.7 ; 62.9$ & & $0.7 ; 27.5$ & & $0.3 ; 9.6$ \\
\hline \multicolumn{8}{|l|}{ Switzerland } \\
\hline $\begin{array}{c}\text { The } \\
\text { Netherlands }\end{array}$ & $0.3 ; 1.7$ & & $0.2 ; 83.8$ & & $0.1 ; 16.2$ & & \\
\hline \multicolumn{8}{|l|}{ Germany } \\
\hline \multicolumn{8}{|l|}{ France } \\
\hline & & \multicolumn{6}{|c|}{ Annual mean } \\
\hline & $\begin{array}{l}\text { Industrial } \\
{\left[\left[\mu \mathrm{g} / \mathrm{m}^{3} ; \% \text { of } \mathrm{PM} \text { mass }\right]\right.}\end{array}$ & $\begin{array}{c}\text { Traffic } \\
{\left[\mu \mathrm{g} / \mathrm{m}^{3} ; \% \text { of Ind }\right]}\end{array}$ & $\begin{array}{c}\text { Urban } \\
{\left[\mu \mathrm{g} / \mathrm{m}^{3} ; \% \text { of }\right.} \\
\text { Ind }]\end{array}$ & \multicolumn{2}{|c|}{$\begin{array}{c}\text { Regional } \\
{\left[\mu \mathrm{g} / \mathrm{m}^{3} ; \% \text { of } \text { Ind }\right]}\end{array}$} & \multicolumn{2}{|c|}{$\begin{array}{c}\text { Continental } \\
{\left[\mu \mathrm{g} / \mathrm{m}^{3} ; \% \text { of Ind }\right]}\end{array}$} \\
\hline & & Anthr. & Anthr. & Natural & Anthr. & Natural & Anthr. \\
\hline Spain & $0.05 ; 0.2$ & & $0.04 ; 79.1$ & & $0.01 ; 13.2$ & & $0.00 ; 7.8$ \\
\hline \multicolumn{8}{|l|}{ Switzerland } \\
\hline $\begin{array}{c}\text { The } \\
\text { Netherlands }\end{array}$ & $2.1 ; 12.7$ & & $0.3 ; 13.6$ & & $2.0 ; 91.3$ & & \\
\hline \multicolumn{8}{|l|}{ Germany } \\
\hline \multicolumn{8}{|l|}{ France } \\
\hline & & \multicolumn{6}{|c|}{ Annual mean } \\
\hline & $\begin{array}{l}\text { Germany } \\
{\left[\mu \mathrm{g} / \mathrm{m}^{3} ; \% \text { of } \mathrm{PM} \text { mass }\right]}\end{array}$ & $\begin{array}{c}\text { Traffic } \\
{\left[\mu \mathrm{g} / \mathrm{m}^{3} ; \%\right]}\end{array}$ & $\begin{array}{c}\text { Urban } \\
{\left[\mu \mathrm{g} / \mathrm{m}^{3} ; \%\right]}\end{array}$ & \multicolumn{2}{|c|}{$\begin{array}{c}\text { Regional } \\
{\left[\mu \mathrm{g} / \mathrm{m}^{3} ; \%\right]}\end{array}$} & \multicolumn{2}{|c|}{$\begin{array}{l}\text { Continental } \\
{\left[\mu \mathrm{g} / \mathrm{m}^{3} ; \%\right]}\end{array}$} \\
\hline & & Anthr. & Anthr. & Natural & Anthr. & Natural & Anthr. \\
\hline Coal_Local & $0.02 ; 0.09$ & $0.0 ; 12.6$ & $0.0 ; 4.9$ & & $0.0 ; 82.5$ & & \\
\hline Coal & $2.3 ; 10.0$ & $0.3 ; 11.4$ & $0.0 ; 0.0$ & & $2.3 ; 98.8$ & & \\
\hline Cooking & $1.1 ; 5.0$ & $0.5 ; 44.3$ & $0.4 ; 32.8$ & & $0.3 ; 22.9$ & & \\
\hline $\begin{array}{l}\text { Photochemi } \\
\text { stry }\end{array}$ & $2.0 ; 8.6$ & $0.1 ; 4.7$ & $0.0 ; 0.3$ & & $1.9 ; 96.9$ & & \\
\hline$S S / R S$ & $0.5 ; 2.0$ & $0.1 ; 20.6$ & $0.3 ; 55.0$ & & $0.1,24.4$ & & \\
\hline \multirow[t]{4}{*}{$\begin{array}{l}\text { Fungal } \\
\text { spores }\end{array}$} & $0.2 ; 0.8$ & & & $0.2 ; 0.8$ & & & \\
\hline & & \multicolumn{6}{|c|}{ Annual mean } \\
\hline & $\begin{array}{l}\text { France } \\
\qquad\left[\mu \mathrm{g} / \mathrm{m}^{3} ; \% \text { of PM mass }\right]\end{array}$ & $\begin{array}{c}\text { Traffic } \\
{\left[\mu \mathrm{g} / \mathrm{m}^{3} ; \%\right]}\end{array}$ & $\begin{array}{c}\text { Urban } \\
{\left[\mu \mathrm{g} / \mathrm{m}^{3} ; \%\right]}\end{array}$ & & $\begin{array}{l}\text { onal } \\
; \%\end{array}$ & $\begin{array}{l}\text { Con } \\
{[\mu \mathrm{g}}\end{array}$ & $\begin{array}{l}\text { ental } \\
; \%]\end{array}$ \\
\hline & & Anthr. & Anthr. & Natural & Anthr. & Natural & Anthr. \\
\hline Marine bio & $1.0 ; 4.8$ & & & $1.0 ; 100$ & & & \\
\hline Land bio & $1.2 ; 5.7$ & & & $1.2 ; 100$ & & & \\
\hline
\end{tabular}

(A) Source contributions calculated for Barcelona (BCN; Spain), Zurich (ZUE; Switzerland), Schiedam (SCH; The Netherlands), Leipzig-Mitte (LMI; Germany) and Lens (LENS; France). 
Table S7: Allocation of PMF source contributions in each country. Mean values for the winter period (DJF) are reported.

\begin{tabular}{|c|c|c|c|c|c|c|c|}
\hline & \multirow{3}{*}{$\begin{array}{c}\text { Source contribution }^{(\mathrm{A})} \\
\text { SIA } \\
{\left[\mu \mathrm{g} / \mathrm{m}^{3} ; \% \text { of PM mass }\right]}\end{array}$} & \multicolumn{6}{|c|}{ Winter mean } \\
\hline & & $\begin{array}{c}\text { Traffic } \\
{\left[\mu \mathrm{g} / \mathrm{m}^{3} ; \% \text { of } \mathrm{SIA}\right]}\end{array}$ & $\begin{array}{c}\text { Urban } \\
{\left[\mu \mathrm{g} / \mathrm{m}^{3} ; \% \text { of }\right.} \\
\mathrm{SIA}]\end{array}$ & \multicolumn{2}{|c|}{$\begin{array}{c}\text { Regional } \\
{\left[\mu \mathrm{g} / \mathrm{m}^{3} ; \% \text { of } \mathrm{SIA}\right]}\end{array}$} & \multicolumn{2}{|c|}{$\begin{array}{c}\text { Continental } \\
{\left[\mu \mathrm{g} / \mathrm{m}^{3} ; \% \text { of } \mathrm{SIA}\right]}\end{array}$} \\
\hline & & Anthr. & Anthr. & Natural & Anthr. & Natural & Anthr. \\
\hline Spain & $8.3 ; 37.0$ & & $3.3 ; 40.0$ & & $3.0 ; 36.1$ & & $1.2 ; 14.8$ \\
\hline Switzerland & $12.2 ; 50.2$ & & $2.5 ; 20.3$ & & $9.8 ; 80.4$ & & \\
\hline $\begin{array}{c}\text { The } \\
\text { Netherlands }\end{array}$ & $18.0 ; 65.1$ & & $3.9 ; 21.6$ & & $14.1 ; 78.4$ & & \\
\hline Germany & $9.9 ; 36.1$ & $1.6 ; 16.0$ & $2.0 ; 20.1$ & & $6.4 ; 65.3$ & & \\
\hline \multirow[t]{4}{*}{ France } & $5.5 ; 30.6$ & & $2.6 ; 47.1$ & & $2.9 ; 52.9$ & & \\
\hline & & \multicolumn{6}{|c|}{ Winter mean } \\
\hline & $\begin{array}{c}\text { SSA } \\
{\left[\mu \mathrm{g} / \mathrm{m}^{3} ; \% \text { of } \mathrm{PM} \text { mass }\right]}\end{array}$ & $\begin{array}{c}\text { Traffic } \\
{\left[\mu \mathrm{g} / \mathrm{m}^{3} ; \% \text { of }\right.} \\
\mathrm{SSA}]\end{array}$ & $\begin{array}{c}\text { Urban } \\
{\left[\mu \mathrm{g} / \mathrm{m}^{3} ; \% \text { of }\right.} \\
\text { SSA }]\end{array}$ & \multicolumn{2}{|c|}{$\begin{array}{c}\text { Regional } \\
{\left[\mu \mathrm{g} / \mathrm{m}^{3} ; \% \text { of } \mathrm{SSA}\right]}\end{array}$} & \multicolumn{2}{|c|}{$\begin{array}{c}\text { Continental } \\
{\left[\mu \mathrm{g} / \mathrm{m}^{3} ; \% \text { of } \mathrm{SSA}\right]}\end{array}$} \\
\hline & & Anthr. & Anthr. & Natural & Anthr. & Natural & Anthr. \\
\hline Spain & $3.3 ; 14.7$ & & $0.8 ; 23.7$ & & $1.6 ; 47.8$ & & $0.9 ; 27.8$ \\
\hline Switzerland & $3.3 ; 13.5$ & & $1.0 ; 32.1$ & & $2.3 ; 70.6$ & & \\
\hline $\begin{array}{c}\text { The } \\
\text { Netherlands }\end{array}$ & $4.2 ; 15.2$ & & $0.3 ; 6.1$ & & $4.0 ; 93.9$ & & \\
\hline \multicolumn{8}{|l|}{ Germany } \\
\hline \multirow[t]{4}{*}{ France } & $2.3 ; 13.1$ & & $0.8 ; 32.6$ & & $1.6 ; 67.4$ & & \\
\hline & & \multicolumn{6}{|c|}{ Winter mean } \\
\hline & $\begin{array}{c}\text { NSA } \\
{\left[\mu \mathrm{g} / \mathrm{m}^{3} ; \% \text { of } \mathrm{PM} \text { mass }\right]}\end{array}$ & $\begin{array}{c}\text { Traffic } \\
{\left[\mu \mathrm{g} / \mathrm{m}^{3} ; \% \text { of }\right.} \\
\mathrm{NSA}]\end{array}$ & $\begin{array}{c}\text { Urban } \\
{\left[\mu \mathrm{g} / \mathrm{m}^{3} ; \% \text { of }\right.} \\
\mathrm{NSA}]\end{array}$ & \multicolumn{2}{|c|}{$\begin{array}{c}\text { Regional } \\
{\left[\mu \mathrm{g} / \mathrm{m}^{3} ; \% \text { of } \mathrm{NSA}\right]}\end{array}$} & \multicolumn{2}{|c|}{$\begin{array}{c}\text { Continental } \\
{\left[\mu \mathrm{g} / \mathrm{m}^{3} ; \% \text { of } \mathrm{NSA}\right]}\end{array}$} \\
\hline & & Anthr. & Anthr. & Natural & Anthr. & Natural & Anthr. \\
\hline Spain & $5.0 ; 22.3$ & & $3.0 ; 60.9$ & & $1.6 ; 32.4$ & & $0.3 ; 6.2$ \\
\hline Switzerland & $8.9 ; 36.8$ & & $1.4 ; 16.0$ & & $7.5 ; 83.9$ & & \\
\hline $\begin{array}{c}\text { The } \\
\text { Netherlands }\end{array}$ & $13.8 ; 50.0$ & & $3.6 ; 26.3$ & & $10.2 ; 73.7$ & & \\
\hline \multicolumn{8}{|l|}{ Germany } \\
\hline \multirow[t]{4}{*}{ France } & $3.1 ; 17.5$ & & $1.8 ; 58.0$ & & $1.3 ; 42.0$ & & \\
\hline & & \multicolumn{6}{|c|}{ Winter mean } \\
\hline & $\begin{array}{c}\text { Mineral } \\
{\left[\mu \mathrm{g} / \mathrm{m}^{3} ; \% \text { of PM mass }\right]}\end{array}$ & $\begin{array}{c}\text { Traffic } \\
{\left[\mu \mathrm{g} / \mathrm{m}^{3} ; \% \text { of }\right.} \\
\text { Mineral] }\end{array}$ & $\begin{array}{c}\text { Urban } \\
{\left[\mu \mathrm{g} / \mathrm{m}^{3} ; \% \text { of }\right.} \\
\text { Mineral] }\end{array}$ & \multicolumn{2}{|c|}{$\begin{array}{c}\text { Regional } \\
{\left[\mu \mathrm{g} / \mathrm{m}^{3} ; \% \text { of Mineral }\right]}\end{array}$} & \multicolumn{2}{|c|}{$\begin{array}{c}\text { Continental } \\
{\left[\mu \mathrm{g} / \mathrm{m}^{3} ; \% \text { of Mineral }\right]}\end{array}$} \\
\hline & & Anthr. & Anthr. & Natural & Anthr. & Natural & Anthr. \\
\hline Spain & $1.7 ; 7.3$ & & $0.7 ; 43.2$ & $0.5 ; 31.5$ & & $0.4 ; 24.3$ & \\
\hline Switzerland & $1.0 ; 4.1$ & & $0.3 ; 28.7$ & $0.7 ; 66.7$ & & & \\
\hline $\begin{array}{c}\text { The } \\
\text { Netherlands }\end{array}$ & $0.3 ; 1.1$ & & $0.16 ; 49.7$ & $0.16 ; 50.3$ & & & \\
\hline Germany & $0.09 ; 0.3$ & $0.0 ; 0.0$ & $0.03 ; 39.9$ & $0.06 ; 68.3$ & & & \\
\hline \multirow[t]{4}{*}{ France } & $3.0 ; 16.7$ & & $1.6 ; 54.1$ & $1.4 ; 45.9$ & & & \\
\hline & & \multicolumn{6}{|c|}{ Winter mean } \\
\hline & $\begin{array}{c}\text { Road traffic } \\
{\left[\mu \mathrm{g} / \mathrm{m}^{3} ; \% \text { of } \mathrm{PM} \text { mass }\right]}\end{array}$ & $\begin{array}{c}\text { Traffic } \\
{\left[\mu \mathrm{g} / \mathrm{m}^{3} ; \% \text { of } \mathrm{RT}\right]}\end{array}$ & $\begin{array}{c}\text { Urban } \\
{\left[\mu \mathrm{g} / \mathrm{m}^{3} ; \% \text { of }\right.} \\
\mathrm{RT}]\end{array}$ & \multicolumn{2}{|c|}{$\begin{array}{c}\text { Regional } \\
{\left[\mu \mathrm{g} / \mathrm{m}^{3} ; \% \text { of } \mathrm{RT}\right]}\end{array}$} & \multicolumn{2}{|c|}{$\begin{array}{c}\text { Continental } \\
{\left[\mu \mathrm{g} / \mathrm{m}^{3} ; \% \text { of } \mathrm{RT}\right]}\end{array}$} \\
\hline & & Anthr. & Anthr. & Natural & Anthr. & Natural & Anthr. \\
\hline Spain & $5.6 ; 25.1$ & & $5.2 ; 92.9$ & & $0.4 ; 7.1$ & & $0.1 ; 1.4$ \\
\hline Switzerland & $3.1 ; 12.8$ & & $2.7 ; 86.5$ & & $0.4 ; 12.9$ & & \\
\hline $\begin{array}{c}\text { The } \\
\text { Netherlands }\end{array}$ & $3.6 ; 13.0$ & & $2.1 ; 59.4$ & & $1.5 ; 40.6$ & & \\
\hline Germany & $5.3 ; 19.2$ & $4.0 ; 76.2$ & $1.1 ; 20.5$ & & $0.3 ; 5.9$ & & \\
\hline France & $1.1 ; 6.3$ & & $0.9 ; 84.0$ & & $0.2 ; 16.0$ & & \\
\hline & & & & Vinter me & & & \\
\hline & $\begin{array}{c}\text { SS } \\
{\left[\mu \mathrm{g} / \mathrm{m}^{3} ; \% \text { of } \mathrm{PM} \text { mass }\right]}\end{array}$ & $\begin{array}{c}\text { Traffic } \\
{\left[\mu \mathrm{g} / \mathrm{m}^{3} ; \% \text { of } \mathrm{SS}\right]}\end{array}$ & $\begin{array}{c}\text { Urban } \\
{\left[\mu \mathrm{g} / \mathrm{m}^{3} ; \% \text { of }\right.} \\
\text { SS] }\end{array}$ & $\begin{array}{r}R \in \\
{\left[\mu \mathrm{g} / \mathrm{m}^{3}\right.}\end{array}$ & of SS] & $\begin{array}{r}\text { Con } \\
{\left[\mu \mathrm{g} / \mathrm{m}^{3}\right.}\end{array}$ & $\begin{array}{l}\text { ental } \\
\text { of } \mathrm{SS}]\end{array}$ \\
\hline & & Anthr. & Anthr. & Natural & Anthr. & Natural & Anthr. \\
\hline Spain & $4.1 ; 18.0$ & & & $4.1 ; 100$ & & & \\
\hline
\end{tabular}




\begin{tabular}{|c|c|c|c|c|c|c|c|}
\hline Switzerland & $2.0 ; 8.4$ & & & \multicolumn{2}{|l|}{$2.0 ; 100$} & & \\
\hline $\begin{array}{c}\text { The } \\
\text { Netherlands }\end{array}$ & $3.5 ; 12.5$ & & & \multicolumn{2}{|l|}{$3.5 ; 100$} & & \\
\hline Germany & $0.9 ; 3.2$ & & & \multicolumn{2}{|l|}{$0.9 ; 100$} & & \\
\hline \multirow[t]{4}{*}{ France } & $3.6 ; 20.1$ & & & \multicolumn{2}{|l|}{$3.6 ; 100$} & & \\
\hline & & \multicolumn{6}{|c|}{ Winter mean } \\
\hline & $\begin{array}{l}\text { Biomass burning } \\
\qquad\left[\mu \mathrm{g} / \mathrm{m}^{3} ; \% \text { of } \mathrm{PM} \text { mass] }\right.\end{array}$ & $\begin{array}{c}\text { Traffic } \\
{\left[\mu \mathrm{g} / \mathrm{m}^{3} ; \% \text { of } \mathrm{BB}\right]}\end{array}$ & $\begin{array}{c}\text { Urban } \\
{\left[\mu \mathrm{g} / \mathrm{m}^{3} ; \% \text { of }\right.} \\
\mathrm{BB}]\end{array}$ & \multicolumn{2}{|c|}{$\begin{array}{c}\text { Regional } \\
{\left[\mu \mathrm{g} / \mathrm{m}^{3} ; \% \text { of } \mathrm{BB}\right]}\end{array}$} & \multicolumn{2}{|c|}{$\begin{array}{c}\text { Continental } \\
{\left[\mu \mathrm{g} / \mathrm{m}^{3} ; \% \text { of } \mathrm{BB}\right]}\end{array}$} \\
\hline & & Anthr. & Anthr. & Natural & Anthr. & Natural & Anthr. \\
\hline \multicolumn{8}{|l|}{ Spain } \\
\hline Switzerland & $5.3 ; 21.9$ & & $1.4 ; 26.8$ & & $3.9 ; 73.1$ & & \\
\hline \multicolumn{8}{|l|}{$\begin{array}{c}\text { The } \\
\text { Netherlands }\end{array}$} \\
\hline Germany & $2.1 ; 7.8$ & $0.0 ; 0.0$ & $0.5 ; 21.2$ & & $1.7 ; 77.8$ & & \\
\hline \multirow[t]{4}{*}{ France } & $4.6 ; 25.7$ & & $2.7 ; 59.4$ & & $1.9 ; 40.6$ & & \\
\hline & & \multicolumn{6}{|c|}{ Winter mean } \\
\hline & $\begin{array}{l}\text { V-Ni } \\
\qquad\left[\mu \mathrm{g} / \mathrm{m}^{3} ; \% \text { of } \mathrm{PM} \text { mass] }\right.\end{array}$ & $\begin{array}{c}\text { Traffic } \\
{\left[\mu \mathrm{g} / \mathrm{m}^{3} ; \% \text { of } \mathrm{V}-\right.} \\
\mathrm{Ni}]\end{array}$ & $\begin{array}{c}\text { Urban } \\
{\left[\mu \mathrm{g} / \mathrm{m}^{3} ; \% \text { of }\right.} \\
\text { V-Ni] }\end{array}$ & \multicolumn{2}{|c|}{$\begin{array}{c}\text { Regional } \\
{\left[\mu \mathrm{g} / \mathrm{m}^{3} ; \% \text { of } \mathrm{V}-\mathrm{Ni}\right]}\end{array}$} & \multicolumn{2}{|c|}{$\begin{array}{c}\text { Continental } \\
{\left[\mu \mathrm{g} / \mathrm{m}^{3} ; \% \text { of } \mathrm{V}-\mathrm{Ni}\right]}\end{array}$} \\
\hline & & Anthr. & Anthr. & Natural & Anthr. & Natural & Anthr. \\
\hline Spain & $1.2 ; 5.3$ & & $0.9 ; 78.8$ & & $0.2 ; 20.9$ & & $0.0 ; 0.4$ \\
\hline \multicolumn{8}{|l|}{ Switzerland } \\
\hline $\begin{array}{c}\text { The } \\
\text { Netherlands }\end{array}$ & $0.5 ; 1.6$ & & $0.4 ; 85.6$ & & $0.1 ; 14.4$ & & \\
\hline \multicolumn{8}{|l|}{ Germany } \\
\hline \multicolumn{8}{|l|}{ France } \\
\hline & & \multicolumn{6}{|c|}{ Winter mean } \\
\hline & $\begin{array}{l}\text { Industrial } \\
\qquad\left[\mu \mathrm{g} / \mathrm{m}^{3} ; \% \text { of } \mathrm{PM} \text { mass] }\right.\end{array}$ & $\begin{array}{c}\text { Traffic } \\
{\left[\mu \mathrm{g} / \mathrm{m}^{3} ; \% \text { of } \mathrm{Ind}\right]}\end{array}$ & $\begin{array}{c}\text { Urban } \\
{\left[\mu \mathrm{g} / \mathrm{m}^{3} ; \% \text { of }\right.} \\
\text { Ind }]\end{array}$ & \multicolumn{2}{|c|}{$\begin{array}{c}\text { Regional } \\
{\left[\mu \mathrm{g} / \mathrm{m}^{3} ; \% \text { of Ind }\right]}\end{array}$} & \multicolumn{2}{|c|}{$\begin{array}{c}\text { Continental } \\
{\left[\mu \mathrm{g} / \mathrm{m}^{3} ; \% \text { of Ind }\right]}\end{array}$} \\
\hline & & Anthr. & Anthr. & Natural & Anthr. & Natural & Anthr. \\
\hline Spain & $0.1 ; 0.3$ & & $0.1 ; 83.7$ & & $0.01 ; 13.9$ & & $0.00 ; 3.5$ \\
\hline \multicolumn{8}{|l|}{ Switzerland } \\
\hline $\begin{array}{c}\text { The } \\
\text { Netherlands }\end{array}$ & $1.8 ; 6.3$ & & $0.1 ; 4.5$ & & $1.7 ; 95.3$ & & \\
\hline \multicolumn{8}{|l|}{ Germany } \\
\hline \multirow{2}{*}{\multicolumn{8}{|c|}{ Winter mean }} \\
\hline & & & & & & & \\
\hline & $\begin{array}{l}\text { Germany } \\
\qquad\left[\mu \mathrm{g} / \mathrm{m}^{3} ; \% \text { of } \mathrm{PM} \text { mass] }\right.\end{array}$ & $\begin{array}{c}\text { Traffic } \\
{\left[\mu \mathrm{g} / \mathrm{m}^{3} ; \% \text { of Ind }\right]}\end{array}$ & $\begin{array}{c}\text { Urban } \\
{\left[\mu \mathrm{g} / \mathrm{m}^{3} ; \% \text { of }\right.} \\
\text { Ind }]\end{array}$ & \multicolumn{2}{|c|}{$\begin{array}{c}\text { Regional } \\
{\left[\mu \mathrm{g} / \mathrm{m}^{3} ; \% \text { of } \mathrm{Ind}\right]}\end{array}$} & $\begin{array}{r}\text { Con } \\
{\left[\mu \mathrm{g} / \mathrm{m}^{3}\right.}\end{array}$ & $\begin{array}{l}\text { ental } \\
\text { of Ind] }\end{array}$ \\
\hline & & Anthr. & Anthr. & Natural & Anthr. & Natural & Anthr. \\
\hline Coal_Local & $0.03 ; 0.12$ & $0.00 ; 6.6$ & $0.00 ; 1.6$ & & $0.03 ; 86.7$ & & \\
\hline Coal & $4.4 ; 15.9$ & $0.5 ; 11.4$ & $0.0 ; 0.0$ & & $3.9 ; 90.0$ & & \\
\hline Cooking & $0.8 ; 2.9$ & $0.3 ; 33.7$ & $0.3 ; 41.1$ & & $0.2 ; 24.7$ & & \\
\hline $\begin{array}{l}\text { Photochemi } \\
\text { stry }\end{array}$ & $1.3 ; 4.7$ & $0.1 ; 5.9$ & $0.0 ; 0.0$ & & $1.2 ; 94.6$ & & \\
\hline$S S / R S$ & $0.5 ; 1.9$ & $0.12 ; 44.8$ & $0.2 ; 31.8$ & & $0.1,28.1$ & & \\
\hline $\begin{array}{l}\text { Fungal } \\
\text { spores }\end{array}$ & $0.0 ; 0.0$ & & & $0.0 ; 0.0$ & & & \\
\hline & & & & Vinter me & & & \\
\hline & $\begin{array}{l}\text { France } \\
{\left[\mu \mathrm{g} / \mathrm{m}^{3} ; \% \text { of } \mathrm{PM} \text { mass] }\right.}\end{array}$ & $\begin{array}{c}\text { Traffic } \\
{\left[\mu \mathrm{g} / \mathrm{m}^{3} ; \%\right]}\end{array}$ & $\begin{array}{c}\text { Urban } \\
{\left[\mu \mathrm{g} / \mathrm{m}^{3} ; \%\right]}\end{array}$ & & $\begin{array}{l}\text { onal } \\
\text { 3; \%] }\end{array}$ & $\begin{array}{l}\text { Con } \\
{[\mu \mathrm{g}}\end{array}$ & $\begin{array}{l}\text { ental } \\
; \%]\end{array}$ \\
\hline & & Anthr. & Anthr. & Natural & Anthr. & Natural & Anthr. \\
\hline Marine bio & $0.1 ; 0.6$ & & & $0.1 ; 100$ & & & \\
\hline Land bio & $0.6 ; 3.6$ & & & $0.6 ; 100$ & & & \\
\hline
\end{tabular}

(A) Source contributions calculated for Barcelona (BCN; Spain), Zurich (ZUE; Switzerland), Schiedam (SCH; The Netherlands), Leipzig-Mitte (LMI; Germany) and Lens (LENS; France). 
Table S8: Allocation of PMF source contributions in each country. Mean values for the summer period (JJA) are reported.

\begin{tabular}{|c|c|c|c|c|c|c|c|}
\hline & \multirow{3}{*}{$\begin{array}{c}\text { Source contribution }^{(A)} \\
\text { SIA } \\
{\left[\mu \mathrm{g} / \mathrm{m}^{3} ; \% \text { of } \mathrm{PM} \text { mass }\right]}\end{array}$} & \multicolumn{6}{|c|}{ Summer mean } \\
\hline & & $\begin{array}{c}\text { Traffic } \\
{\left[\mu \mathrm{g} / \mathrm{m}^{3} ; \% \text { of } \mathrm{SIA}\right]}\end{array}$ & $\begin{array}{c}\text { Urban } \\
{\left[\mu \mathrm{g} / \mathrm{m}^{3} ; \% \text { of }\right.} \\
\mathrm{SIA}]\end{array}$ & \multicolumn{2}{|c|}{$\begin{array}{c}\text { Regional } \\
{\left[\mu \mathrm{g} / \mathrm{m}^{3} ; \% \text { of } \mathrm{SIA}\right]}\end{array}$} & \multicolumn{2}{|c|}{$\begin{array}{c}\text { Continental } \\
{\left[\mu \mathrm{g} / \mathrm{m}^{3} ; \% \text { of } \mathrm{SIA}\right]}\end{array}$} \\
\hline & & Anthr. & Anthr. & Natural & Anthr. & Natural & Anthr. \\
\hline Spain & $7.2 ; 27.7$ & & $0.9 ; 11.9$ & & $1.3 ; 18.7$ & & $5.0 ; 69.4$ \\
\hline Switzerland & $5.4 ; 39.5$ & & $1.2 ; 22.7$ & & $4.1 ; 76.5$ & & \\
\hline $\begin{array}{c}\text { The } \\
\text { Netherlands }\end{array}$ & $6.2 ; 51.9$ & & $1.3 ; 20.6$ & & $4.9 ; 79.4$ & & \\
\hline Germany & $2.1 ; 11.4$ & $0.2 ; 8.5$ & $0.6 ; 28.5$ & & $1.3 ; 61.8$ & & \\
\hline \multirow[t]{4}{*}{ France } & $3.3 ; 16.3$ & & $1.3 ; 39.6$ & & $2.0 ; 60.0$ & & \\
\hline & & \multicolumn{6}{|c|}{ Summer mean } \\
\hline & $\begin{array}{c}\text { SSA } \\
{\left[\mu \mathrm{g} / \mathrm{m}^{3} ; \% \text { of } \mathrm{PM} \text { mass }\right]}\end{array}$ & $\begin{array}{c}\text { Traffic } \\
{\left[\mu \mathrm{g} / \mathrm{m}^{3} ; \% \text { of }\right.} \\
\text { SSA }]\end{array}$ & $\begin{array}{c}\text { Urban } \\
{\left[\mu \mathrm{g} / \mathrm{m}^{3} ; \% \text { of }\right.} \\
\mathrm{SSA}]\end{array}$ & \multicolumn{2}{|c|}{$\begin{array}{c}\text { Regional } \\
{\left[\mu \mathrm{g} / \mathrm{m}^{3} ; \% \text { of } \mathrm{SSA}\right]}\end{array}$} & \multicolumn{2}{|c|}{$\begin{array}{c}\text { Continental } \\
{\left[\mu \mathrm{g} / \mathrm{m}^{3} ; \% \text { of } \mathrm{SSA}\right]}\end{array}$} \\
\hline & & Anthr. & Anthr. & Natural & Anthr. & Natural & Anthr. \\
\hline Spain & $6.2 ; 23.7$ & & $0.6 ; 9.5$ & & $1.2 ; 19.2$ & & $4.4 ; 71.3$ \\
\hline Switzerland & $5.2 ; 37.7$ & & $1.0 ; 20.1$ & & $4.1 ; 78.5$ & & \\
\hline $\begin{array}{c}\text { The } \\
\text { Netherlands }\end{array}$ & $2.9 ; 24.3$ & & $0.3 ; 17.8$ & & $2.6 ; 82.2$ & & \\
\hline \multicolumn{8}{|l|}{ Germany } \\
\hline \multirow[t]{4}{*}{ France } & $2.5 ; 12.5$ & & $0.6 ; 21.9$ & & $1.9 ; 77.3$ & & \\
\hline & & \multicolumn{6}{|c|}{ Summer mean } \\
\hline & $\begin{array}{c}\text { NSA } \\
{\left[\mu \mathrm{g} / \mathrm{m}^{3} ; \% \text { of } \mathrm{PM} \text { mass }\right]}\end{array}$ & $\begin{array}{c}\text { Traffic } \\
{\left[\mu \mathrm{g} / \mathrm{m}^{3} ; \% \text { of }\right.} \\
\mathrm{NSA}]\end{array}$ & $\begin{array}{c}\text { Urban } \\
{\left[\mu \mathrm{g} / \mathrm{m}^{3} ; \% \text { of }\right.} \\
\mathrm{NSA}]\end{array}$ & \multicolumn{2}{|c|}{$\begin{array}{c}\text { Regional } \\
{\left[\mu \mathrm{g} / \mathrm{m}^{3} ; \% \text { of } \mathrm{NSA}\right]}\end{array}$} & \multicolumn{2}{|c|}{$\begin{array}{c}\text { Continental } \\
{\left[\mu \mathrm{g} / \mathrm{m}^{3} ; \% \text { of } \mathrm{NSA}\right]}\end{array}$} \\
\hline & & Anthr. & Anthr. & Natural & Anthr. & Natural & Anthr. \\
\hline Spain & $1.0 ; 4.0$ & & $0.3 ; 26.1$ & & $0.2 ; 15.8$ & & $0.6 ; 58.1$ \\
\hline Switzerland & $0.3 ; 1.9$ & & $0.2 ; 76.5$ & & $0.1 ; 33.5$ & & \\
\hline $\begin{array}{c}\text { The } \\
\text { Netherlands }\end{array}$ & $3.3 ; 27.5$ & & $0.8 ; 23.1$ & & $2.5 ; 76.9$ & & \\
\hline \multicolumn{8}{|l|}{ Germany } \\
\hline \multirow[t]{4}{*}{ France } & $0.8 ; 3.9$ & & $0.8 ; 100.0$ & & $0.0 ; 0.0$ & & \\
\hline & & \multicolumn{6}{|c|}{ Summer mean } \\
\hline & $\begin{array}{c}\text { Mineral } \\
{\left[\mu \mathrm{g} / \mathrm{m}^{3} ; \% \text { of } \mathrm{PM} \text { mass }\right]}\end{array}$ & $\begin{array}{c}\text { Traffic } \\
{\left[\mu \mathrm{g} / \mathrm{m}^{3} ; \% \text { of }\right.} \\
\text { Mineral] }\end{array}$ & $\begin{array}{c}\text { Urban } \\
{\left[\mu \mathrm{g} / \mathrm{m}^{3} ; \% \text { of }\right.} \\
\text { Mineral] }\end{array}$ & \multicolumn{2}{|c|}{$\begin{array}{c}\text { Regional } \\
{\left[\mu \mathrm{g} / \mathrm{m}^{3} ; \% \text { of Mineral] }\right.}\end{array}$} & \multicolumn{2}{|c|}{$\begin{array}{c}\text { Continental } \\
{\left[\mu \mathrm{g} / \mathrm{m}^{3} ; \% \text { of Mineral }\right]}\end{array}$} \\
\hline & & Anthr. & Anthr. & Natural & Anthr. & Natural & Anthr. \\
\hline Spain & $3.9 ; 15.0$ & & $0.7 ; 18.0$ & $0.1 ; 1.4$ & & $3.2 ; 80.6$ & \\
\hline Switzerland & $3.2 ; 23.1$ & & $0.9 ; 28.4$ & $2.3 ; 72.1$ & & & \\
\hline $\begin{array}{c}\text { The } \\
\text { Netherlands }\end{array}$ & $0.3 ; 2.7$ & & $0.02 ; 6.0$ & $0.3 ; 94.0$ & & & \\
\hline Germany & $1.1 ; 5.8$ & $0.0 ; 0.0$ & $1.1 ; 92.0$ & $0.0 ; 8.0$ & & & \\
\hline \multirow[t]{4}{*}{ France } & $3.3 ; 16.3$ & & $0.3 ; 8.9$ & $3.0 ; 90.1$ & & & \\
\hline & & \multicolumn{6}{|c|}{ Summer mean } \\
\hline & $\begin{array}{c}\text { Road traffic } \\
{\left[\mu \mathrm{g} / \mathrm{m}^{3} ; \% \text { of } \mathrm{PM} \text { mass }\right]}\end{array}$ & $\begin{array}{c}\text { Traffic } \\
{\left[\mu \mathrm{g} / \mathrm{m}^{3} ; \% \text { of } \mathrm{RT}\right]}\end{array}$ & $\begin{array}{c}\text { Urban } \\
{\left[\mu \mathrm{g} / \mathrm{m}^{3} ; \% \text { of }\right.} \\
\mathrm{RT}]\end{array}$ & \multicolumn{2}{|c|}{$\begin{array}{c}\text { Regional } \\
{\left[\mu \mathrm{g} / \mathrm{m}^{3} ; \% \text { of } \mathrm{RT}\right]}\end{array}$} & \multicolumn{2}{|c|}{$\begin{array}{c}\text { Continental } \\
{\left[\mu \mathrm{g} / \mathrm{m}^{3} ; \% \text { of } \mathrm{RT}\right]}\end{array}$} \\
\hline & & Anthr. & Anthr. & Natural & Anthr. & Natural & Anthr. \\
\hline Spain & $2.9 ; 11.0$ & & $2.5 ; 86.5$ & & $0.3 ; 11.7$ & & $0.1 ; 1.8$ \\
\hline Switzerland & $2.8 ; 20.4$ & & $2.4 ; 86.6$ & & $0.4 ; 14.5$ & & \\
\hline $\begin{array}{c}\text { The } \\
\text { Netherlands }\end{array}$ & $0.6 ; 4.8$ & & $0.5 ; 88.8$ & & $0.0 ; 11.2$ & & \\
\hline Germany & $5.1 ; 27.5$ & $3.4 ; 66.2$ & $1.3 ; 25.0$ & & $0.5 ; 8.9$ & & \\
\hline \multirow[t]{4}{*}{ France } & $1.3 ; 6.3$ & & $1.0 ; 77.9$ & & $0.3 ; 23.2$ & & \\
\hline & & & & ummer me & & & \\
\hline & $\begin{array}{c}\text { SS } \\
{\left[\mu \mathrm{g} / \mathrm{m}^{3} ; \% \text { of } \mathrm{PM} \text { mass }\right]}\end{array}$ & $\begin{array}{c}\text { Traffic } \\
{\left[\mu \mathrm{g} / \mathrm{m}^{3} ; \% \text { of } \mathrm{SS}\right]}\end{array}$ & $\begin{array}{c}\text { Urban } \\
{\left[\mu \mathrm{g} / \mathrm{m}^{3} ; \% \text { of }\right.} \\
\mathrm{SS}]\end{array}$ & $\begin{array}{r}R e \\
{\left[\mu g / m^{3}\right.}\end{array}$ & $\begin{array}{l}\text { nal } \\
\text { of SS] }\end{array}$ & $\begin{array}{r}\text { Cor } \\
{\left[\mu \mathrm{g} / \mathrm{m}^{3}\right.}\end{array}$ & $\begin{array}{l}\text { ental } \\
6 \text { of SS] }\end{array}$ \\
\hline & & Anthr. & Anthr. & Natural & Anthr. & Natural & Anthr. \\
\hline Spain & $6.9 ; 26.5$ & & & $6.9 ; 100$ & & & \\
\hline
\end{tabular}




\begin{tabular}{|c|c|c|c|c|c|c|c|}
\hline Switzerland & $2.5 ; 18.1$ & & & \multicolumn{2}{|l|}{$2.5 ; 100$} & & \\
\hline $\begin{array}{c}\text { The } \\
\text { Netherlands }\end{array}$ & $0.8 ; 6.7$ & & & \multicolumn{2}{|l|}{$0.8 ; 100$} & & \\
\hline Germany & $0.9 ; 5.1$ & & & \multicolumn{2}{|l|}{$0.9 ; 100$} & & \\
\hline \multirow[t]{4}{*}{ France } & $6.4 ; 31.5$ & & & \multicolumn{2}{|l|}{$6.4 ; 100$} & & \\
\hline & & \multicolumn{6}{|c|}{ Summer mean } \\
\hline & $\begin{array}{l}\text { Biomass burning } \\
{\left[\mu \mathrm{g} / \mathrm{m}^{3} ; \% \text { of } \mathrm{PM} \text { mass] }\right.}\end{array}$ & $\begin{array}{c}\text { Traffic } \\
{\left[\mu \mathrm{g} / \mathrm{m}^{3} ; \% \text { of BB] }\right.}\end{array}$ & $\begin{array}{c}\text { Urban } \\
{\left[\mu \mathrm{g} / \mathrm{m}^{3} ; \% \text { of }\right.} \\
\mathrm{BB}]\end{array}$ & \multicolumn{2}{|c|}{$\begin{array}{c}\text { Regional } \\
{\left[\mu \mathrm{g} / \mathrm{m}^{3} ; \% \text { of } \mathrm{BB}\right]}\end{array}$} & \multicolumn{2}{|c|}{$\begin{array}{c}\text { Continental } \\
{\left[\mu \mathrm{g} / \mathrm{m}^{3} ; \% \text { of } \mathrm{BB}\right]}\end{array}$} \\
\hline & & Anthr. & Anthr. & Natural & Anthr. & Natural & Anthr. \\
\hline \multicolumn{8}{|l|}{ Spain } \\
\hline Switzerland & $0.3 ; 2.2$ & & $0.02 ; 7.8$ & & $0.3 ; 88.2$ & & \\
\hline \multicolumn{8}{|l|}{$\begin{array}{c}\text { The } \\
\text { Netherlands }\end{array}$} \\
\hline Germany & $0.5 ; 2.8$ & $0.0 ; 0.0$ & $0.5 ; 96.2$ & & $0.0 ; 3.8$ & & \\
\hline \multirow[t]{4}{*}{ France } & $0.0 ; 0.0$ & & $0.0 ; 0.0$ & & $0.0 ; 0.0$ & & \\
\hline & & \multicolumn{6}{|c|}{ Summer mean } \\
\hline & $\begin{array}{l}\text { V-Ni } \\
\qquad\left[\mu \mathrm{g} / \mathrm{m}^{3} ; \% \text { of PM mass] }\right.\end{array}$ & $\begin{array}{c}\text { Traffic } \\
{\left[\mu \mathrm{g} / \mathrm{m}^{3} ; \% \text { of } \mathrm{V}-\right.} \\
\mathrm{Ni}]\end{array}$ & $\begin{array}{c}\text { Urban } \\
{\left[\mu \mathrm{g} / \mathrm{m}^{3} ; \% \text { of }\right.} \\
\text { V-Ni] }\end{array}$ & \multicolumn{2}{|c|}{$\begin{array}{c}\text { Regional } \\
{\left[\mu \mathrm{g} / \mathrm{m}^{3} ; \% \text { of } \mathrm{V}-\mathrm{Ni}\right]}\end{array}$} & \multicolumn{2}{|c|}{$\begin{array}{c}\text { Continental } \\
{\left[\mu \mathrm{g} / \mathrm{m}^{3} ; \% \text { of } \mathrm{V}-\mathrm{Ni}\right]}\end{array}$} \\
\hline & & Anthr. & Anthr. & Natural & Anthr. & Natural & Anthr. \\
\hline Spain & $3.4 ; 13.2$ & & $1.9 ; 56.4$ & & $1.0 ; 30.3$ & & $0.5 ; 13.4$ \\
\hline \multicolumn{8}{|l|}{ Switzerland } \\
\hline $\begin{array}{c}\text { The } \\
\text { Netherlands }\end{array}$ & $0.3 ; 2.9$ & & $0.3 ; 83.0$ & & $0.1 ; 17.0$ & & \\
\hline \multicolumn{8}{|l|}{ Germany } \\
\hline \multicolumn{8}{|l|}{ France } \\
\hline & & \multicolumn{6}{|c|}{ Summer mean } \\
\hline & $\begin{array}{l}\text { Industrial } \\
\qquad\left[\mu \mathrm{g} / \mathrm{m}^{3} ; \% \text { of } \mathrm{PM} \text { mass }\right]\end{array}$ & $\begin{array}{c}\text { Traffic } \\
{\left[\mu \mathrm{g} / \mathrm{m}^{3} ; \% \text { of Ind }\right]}\end{array}$ & $\begin{array}{c}\text { Urban } \\
{\left[\mu \mathrm{g} / \mathrm{m}^{3} ; \% \text { of }\right.} \\
\text { Ind }]\end{array}$ & \multicolumn{2}{|c|}{$\begin{array}{c}\text { Regional } \\
{\left[\mu \mathrm{g} / \mathrm{m}^{3} ; \% \text { of Ind] }\right.}\end{array}$} & \multicolumn{2}{|c|}{$\begin{array}{c}\text { Continental } \\
{\left[\mu \mathrm{g} / \mathrm{m}^{3} ; \% \text { of Ind }\right]}\end{array}$} \\
\hline & & Anthr. & Anthr. & Natural & Anthr. & Natural & Anthr. \\
\hline Spain & $0.03 ; 0.1$ & & $0.03 ; 81.5$ & & $0.00 ; 11.6$ & & $0.00 ; 6.9$ \\
\hline \multicolumn{8}{|l|}{ Switzerland } \\
\hline $\begin{array}{c}\text { The } \\
\text { Netherlands }\end{array}$ & $2.4 ; 19.9$ & & $0.1 ; 3.4$ & & $2.3 ; 95.7$ & & \\
\hline \multicolumn{8}{|l|}{ Germany } \\
\hline \multicolumn{8}{|l|}{ France } \\
\hline & & \multicolumn{6}{|c|}{ Summer mean } \\
\hline & $\begin{array}{l}\text { Germany } \\
\qquad\left[\mu \mathrm{g} / \mathrm{m}^{3} ; \% \text { of } \mathrm{PM} \text { mass] }\right.\end{array}$ & $\begin{array}{c}\text { Traffic } \\
{\left[\mu \mathrm{g} / \mathrm{m}^{3} ; \% \text { of Ind }\right]}\end{array}$ & $\begin{array}{c}\text { Urban } \\
{\left[\mu \mathrm{g} / \mathrm{m}^{3} ; \% \text { of }\right.} \\
\text { Ind }]\end{array}$ & $\begin{array}{r}\mathrm{Re} \\
{\left[\mu \mathrm{g} / \mathrm{m}^{3}\right.}\end{array}$ & of Ind] & $\begin{array}{r}\text { Con } \\
{\left[\mu \mathrm{g} / \mathrm{m}^{3}\right.}\end{array}$ & $\begin{array}{l}\text { ental } \\
6 \text { of Ind] }\end{array}$ \\
\hline & & Anthr. & Anthr. & Natural & Anthr. & Natural & Anthr. \\
\hline Coal_Local & $0.01 ; 0.03$ & $0.00 ; 73.7$ & $0.00 ; 0.0$ & & $0.00 ; 24.1$ & & \\
\hline Coal & $0.03 ; 0.2$ & $0.02 ; 67.1$ & $0.0 ; 0.0$ & & $0.01 ; 33.3$ & & \\
\hline Cooking & $1.5 ; 8.2$ & $1.0 ; 65.1$ & $0.5 ; 31.0$ & & $0.0 ; 4.0$ & & \\
\hline $\begin{array}{l}\text { Photochemi } \\
\text { stry }\end{array}$ & $2.7 ; 14.6$ & $0.2 ; 7.4$ & $0.3 ; 10.1$ & & $2.2 ; 83.0$ & & \\
\hline$S S / R S$ & $0.5 ; 2.4$ & $0.0 ; 8.0$ & $0.5 ; 91.0$ & & $0.0,0.0$ & & \\
\hline $\begin{array}{l}\text { Fungal } \\
\text { spores }\end{array}$ & $0.4 ; 2.0$ & & & $0.0 ; 0.0$ & & & \\
\hline & & & & ummer me & & & \\
\hline & $\begin{array}{l}\text { France } \\
\qquad\left[\mu \mathrm{g} / \mathrm{m}^{3} ; \% \text { of } \mathrm{PM} \text { mass] }\right.\end{array}$ & $\begin{array}{c}\text { Traffic } \\
{\left[\mu \mathrm{g} / \mathrm{m}^{3} ; \%\right]}\end{array}$ & $\begin{array}{c}\text { Urban } \\
{\left[\mu \mathrm{g} / \mathrm{m}^{3} ; \%\right]}\end{array}$ & & $\begin{array}{l}\text { nal } \\
; \%]\end{array}$ & $\begin{array}{l}\text { Con } \\
{[\mu \mathrm{g}}\end{array}$ & $\begin{array}{l}\text { ental } \\
3 ; \%]\end{array}$ \\
\hline & & Anthr. & Anthr. & Natural & Anthr. & Natural & Anthr. \\
\hline Marine bio & $3.8 ; 18.4$ & & & $3.8 ; 100$ & & & \\
\hline Land bio & $2.4 ; 12.0$ & & & $2.4 ; 100$ & & & \\
\hline
\end{tabular}

(A) Source contributions calculated for Barcelona (BCN; Spain), Zurich (ZUE; Switzerland), Schiedam (SCH; The Netherlands), Leipzig-Mitte (LMI; Germany) and Lens (LENS; France). 